\title{
A Dynamical Mechanism for Secondary Eyewall Formation in Tropical Cyclones
}

\author{
YOSHIAKI MIYAMOTO ${ }^{\mathrm{a}}$ \\ Rosenstiel School of Marine and Atmospheric Science, University of Miami, Miami, Florida, and RIKEN Advanced Institute \\ for Computational Science, Kobe, Japan \\ DAVID S. NOLAN \\ Rosenstiel School of Marine and Atmospheric Science, University of Miami, Miami, Florida \\ NORIHIKO SUGIMOTO \\ Department of Physics, Keio University, Kanagawa, Japan
}

(Manuscript received 3 February 2018, in final form 25 June 2018)

\begin{abstract}
This study proposes that secondary eyewall formation (SEF) of tropical cyclones (TCs) can be attributed to an instability of flow in the free atmosphere coupled with Ekman pumping. Unstable solutions of a 1.5-layer shallow-water system are obtained under fast-wind speed conditions in the free atmosphere. The instability condition derived in the linear model indicates the importance of the ratio of angular velocity to vorticity, and the condition is more likely to be satisfied when the ratio is large and its radial gradient is positive. Thus, fast angular velocity, low absolute vertical vorticity, small negative radial gradient of angular velocity, and large negative gradient of vertical vorticity are favorable. Eigenvalue analyses are performed over a wide range of parameters using a vorticity profile with an infinitesimal secondary maximum. The growth rate increases with vorticity outside the radius of maximum wind (RMW), the radius of the secondary vorticity maximum, its magnitude, and the Rossby number defined by maximum tangential velocity, the RMW, and the Coriolis parameter. Furthermore, the growth rate is positive only between 2 and 7 times the RMW, and it is negative close to or far outside the RMW. These features are consistent with previous studies on SEF. A dimensionless quantity $\Upsilon$ obtained from the unstable condition in the linear theory is applied to SEF events simulated by two different full-physics numerical models; $\Upsilon$ increases several hours before a secondary peak of tangential velocity forms, suggesting that the initial process of SEF can be attributed to the proposed mechanism.
\end{abstract}

\section{Introduction}

Strong tropical cyclones (TCs) often experience secondary eyewall formation (SEF; Willoughby et al. 1982), in which a new eyewall forms outside the original eyewall. It is usually followed by an eyewall replacement cycle, and hence, predicting SEF is important for accurate TC forecasts. However, there is no widely accepted theory for SEF despite the significant effort by many researches, especially in the last decade. We here present a new theory focusing on flow interaction between the boundary layer and the free atmosphere.

\footnotetext{
${ }^{a}$ Current affiliation: Faculty of Environment and Information Studies, Keio University, Kanagawa, Japan.
}

Corresponding author: Yoshiaki Miyamoto, ymiya@sfc.keio.ac.jp
SEF often occurs at a radius of 2-4 times the radius of maximum wind (RMW) in recently intensified TCs (Willoughby et al. 1982; Black and Willoughby 1992; Dodge et al. 1999; Houze et al. 2007; Kossin and Sitkowski 2009; Kuo et al. 2009; Wu et al. 2016). The key parameters and processes for SEF proposed by previous studies are inertial instability (Willoughby et al. 1982), terrain effect (Hawkins 1983), ice microphysics (Willoughby et al. 1984), inflow surge (Molinari and Skubis 1985; Molinari and Vollaro 1989), vortex Rossby waves (Montgomery and Kallenbach 1997; Terwey and Montgomery 2003), potential-vorticity intrusion from upper layer (Nong and Emanuel 2003), axisymmetrization of vorticity disturbance (Kuo et al. 2004, 2008), the rectification of eddies in the $\beta$ skirt outside the primary eyewall (Terwey and Montgomery 2008), supergradient wind (Huang et al. 2012; Wu et al. 2012), wind field 
expansion (Rozoff et al. 2012; Sun et al. 2013), radial vorticity gradient outside the RMW where relative vorticity is low (Kepert 2013), top-down process associated with interaction with upper-level trough (Leroux et al. 2013), rainband heating (Zhu and Zhu 2014; Zhang et al. 2016), and upper-level outflow interactions (Dai et al. 2017).

Kepert (2013) showed the importance of the radial gradient of relative vorticity in a low-vorticity environment using both linear and nonlinear boundary layer models. According to the Ekman theory for a rotating fluid, Ekman pumping velocity is proportional to the radial vorticity gradient and inversely proportional to the square of the absolute vorticity itself. Kepert (2013) and Kepert and Nolan (2014) proposed a positive feedback that can lead to SEF: when the radial gradient of vertical relative vorticity is steep, Ekman pumping velocity is large, the upward velocity is enhanced by convection and causes further convergence, and the vorticity gradient steepens. However, the previous studies consider vorticity profiles after SEF and secondary wind maximum, in which a secondary peak of tangential velocity is already present outside the RMW. From ensembles of numerical simulations, Zhang et al. (2016) showed that the diabatic heating outside the RMW in the upper layer proceeds downward, changing the radial vorticity profile, and the secondary eyewall develops through the interaction between convection and boundary layer processes. Although the interaction might work once the vorticity gradient is steep, the proposed mechanism is incomplete because it offers no explanation of conditions under which outer rainbands produce such a strong vorticity gradient (otherwise, rainbands would always cause SEF). Therefore, the underlying mechanism of $\mathrm{SEF}$, especially for the initial process (i.e., when and where SEF occurs), is unclear.

This study proposes a linear instability via an interaction between Ekman pumping and the flow in the free atmosphere, which is not associated with moist convection, unlike the previous theories, and hypothesizes that very early processes of SEF are attributed to this instability. Once the initial processes of SEF proposed in this study create a local maximum of vertical velocity or tangential velocity outside the primary eyewall, the heating-convergence feedback might work to develop the secondary eyewall, followed by an eyewall replacement cycle (Kepert 2013; Kepert and Nolan 2014).

The rest of the paper is organized as follows. We develop a linear theory for a 1.5-layer shallow-water system in Cartesian coordinates and eigenvalue analyses and numerical integrations for a nonlinear equation set

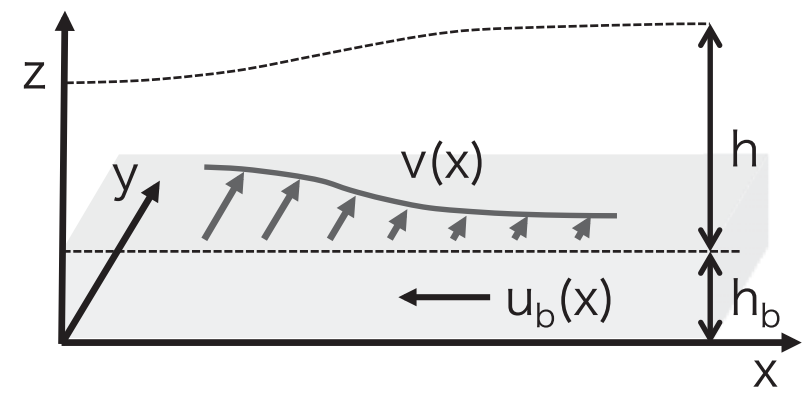

FIG. 1. Schematic of the one-dimensional, 1.5-layer fluid system on the $f$ plane. The system consists of free atmosphere with a depth of $h$ and boundary layer with $h_{b}$.

are conducted in section 2. The theory is extended to cylindrical coordinates and a condition for the instability to occur is derived and linear stability analyses of an axisymmetric 1.5-layer shallow-water system are performed in section 3 . The unstable condition is tested by applying it to simulation results by two nonhydrostatic full-physics models in section 4 . The results are discussed by comparing them with previous studies in section 5. This study is concluded in section 6 .

\section{Extension of the Ekman theory in Cartesian coordinates to high-wind speed conditions}

\section{a. Classic Ekman theory}

Ekman pumping is a vertical motion at the top of the planetary boundary layer induced by surface friction (Ekman 1905; Holton 1992). A cyclonic flow with Ekman pumping transports angular momentum in the interior layer outward, and the flow decays much more quickly than would occur from viscous diffusion (vortex spindown; Holton 1992; Eliassen and Lystad 1977). ${ }^{1}$ This can be explained by considering a one-dimensional 1.5-layer shallow-water system on an $f$ plane with a semislip lower-boundary condition: a free atmosphere plus a boundary layer (cf. Fig. 1, but velocity in the free atmosphere is weak and its horizontal variations are slow). We can obtain an analytical solution for the evolution of perturbations of relative vorticity, and the solution indicates that the vorticity perturbation decays exponentially. A key relationship in the classic Ekman theory is that perturbation vertical velocity at the top of boundary layer $w^{\prime}$ is proportional to the horizontal gradient of the $y$-velocity perturbation $\partial_{x} v^{\prime}$ :

\footnotetext{
${ }^{1} \mathrm{We}$ here consider the atmosphere in which the interior layer is located above the boundary layer. The basic concept of the present theory can be applied to the ocean and other flows.
} 


$$
w^{\prime} \propto \frac{\partial v^{\prime}}{\partial x} .
$$

Details to obtain this relationship in the 1.5-layer shallow-water system can be found in appendix A.

The Ekman damping process for velocity perturbations can be interpreted as follows: once a positive $w^{\prime}$ is added at the top of the boundary layer (left-hand side of Fig. 2), it produces positive $x$-velocity perturbation $u^{\prime}$ in the free atmosphere for mass conservation at larger $x$ and negative $u^{\prime}$ at smaller $x$, which decelerates and accelerates, respectively, $y$-velocity perturbation $v^{\prime}$ by transporting basic-state absolute vorticity. Thus, the horizontal gradient of perturbation $y$ velocity $\partial_{x} v^{\prime}$ is negative around the $w^{\prime}$ peak. Assuming that $v^{\prime}$ in the boundary layer is changed in a similar way, by mass divergence, $w^{\prime}$ tends to be reduced, and hence, the initially added $w^{\prime}$ is damped. This is because of (1).

The classic Ekman theory is widely accepted and has provided many insights into geophysical fluid dynamics. For example, the effects of Ekman pumping on Rossby waves are modeled by adding vertical velocity at the top of the boundary layer that is proportional to vertical relative vorticity (e.g., James 1995; Vallis 2017). Although the theory can capture fundamental processes for various phenomena, the assumptions may limit its application. In particular, it is assumed that the background flow is not fast and its spatial gradient (relative vorticity) is not large. Hence, the theory may not be applicable to some circumstances such as jets or tropical cyclones in which the horizontal velocity is fast in the lower free atmosphere.

\section{b. Extension of Ekman theory to fast velocity conditions}

We here hypothesize that the relationship in (1) can possibly be reversed when we include the advection in the boundary layer. If this were to occur, $w^{\prime}$ would be negatively proportional to the gradient of $v^{\prime}$; that is, $w^{\prime} \propto-\partial_{x} v^{\prime}$ (right-hand side of Fig. 2). According to the mechanism described above, a negative gradient of $\boldsymbol{v}^{\prime}$ is produced around a positive peak of $w^{\prime}$ because of mass conservation and horizontal transport of absolute vorticity, which is invariant even in fast flow conditions. In this case, however, the negative velocity gradient tends to produce positive $w^{\prime}$ around the initial peak. It means that $w^{\prime}$ is amplified and thus perturbations will grow exponentially.

To demonstrate the hypothesized instability, equations for the 1.5-layer shallow-water system on an $f$ plane [(A7)-(A11)] are linearized. The basic state satisfies the geostrophic balance in the free atmosphere. In addition, we here assume that the basic-state $y$ velocity $\bar{v}$ is strong so that the advection of $v^{\prime}$ by basic state $x$ velocity $\bar{u}_{b}$

\section{Classic theory

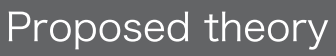

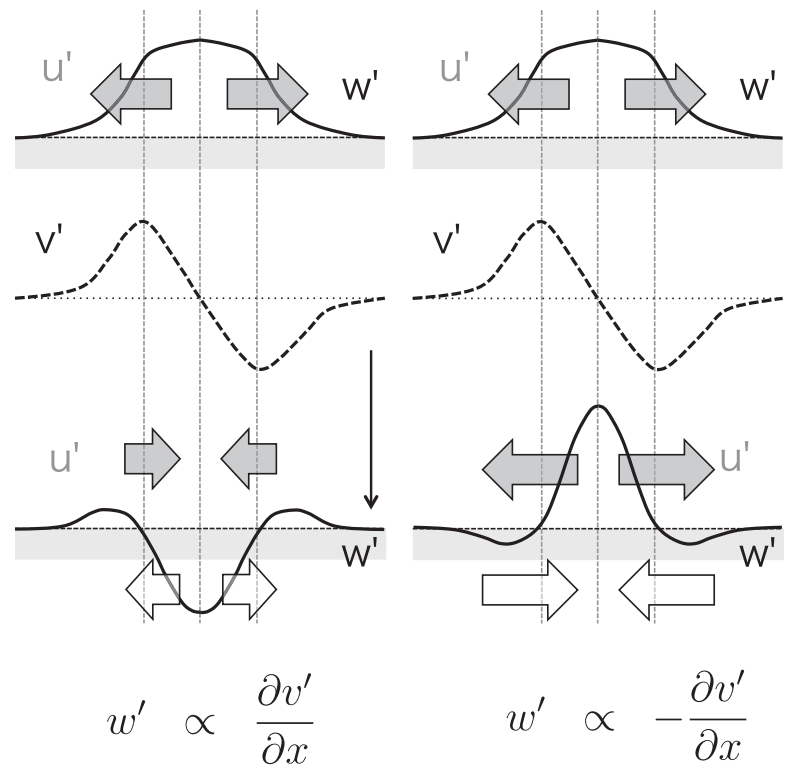

FIG. 2. Schematic of the spatial profiles of velocity disturbances that respond to (top) the initial perturbation of $w^{\prime}$ in the (left) classic theory and (right) proposed theory. The bold and dashed lines stand for $w^{\prime}$ at the top of boundary layer and $v^{\prime}$. The gray and white thick arrows indicate $u^{\prime}$ in the free atmosphere and in the boundary layer.

in the boundary layer is nonnegligible. A diagnostic equation for $\bar{u}_{b}$ is obtained from the momentum equation for the $y$ direction $[(\mathrm{A} 11)]$ as

$$
\bar{u}_{b}=-\frac{|\overline{\boldsymbol{v}}| \overline{\boldsymbol{v}}}{\bar{\zeta}_{a} h_{b}} .
$$

The linearized equations for mass and momentum conservation in the free atmosphere are

$$
\begin{aligned}
\frac{1}{\operatorname{Ro}_{1}} \frac{\partial h^{\prime}}{\partial t} & =-\frac{\partial u^{\prime} \bar{h}}{\partial x}+w^{\prime}, \\
\frac{\operatorname{Ro}}{\operatorname{Fr}^{2}} \frac{\partial h^{\prime}}{\partial x} & =v^{\prime}, \\
\frac{1}{G_{1}} \frac{\partial v^{\prime}}{\partial t} & =-\bar{\zeta}_{a} u^{\prime},
\end{aligned}
$$

and those in the boundary layer are

$$
\begin{gathered}
-\frac{\partial h_{b} u_{b}^{\prime}}{\partial x}=w^{\prime}, \\
-u_{b}^{\prime} \bar{\zeta}_{a}-\operatorname{Ro}_{b} \frac{\partial v^{\prime}}{\partial x}=\frac{2|\bar{v}|}{h_{b}} v^{\prime}
\end{gathered}
$$


We have neglected correlation terms between perturbations (e.g., $\left.u^{\prime} h^{\prime}\right)$. The fundamental differences from the classic Ekman theory in the present 1.5-layer system are the advection of $v^{\prime}$ by $\bar{u}_{b}$ [the second term in the lhs of (7)] and the nonuniform basic-state $y$-velocity $\left[v^{\prime}=\bar{v}(x)\right.$, and hence, $\left.\bar{\zeta}_{a}(x)=\operatorname{Ro} \partial_{x} \bar{v}+1\right]$, the first term in the lhs of (7) and the rhs of (5).

Substituting (7) into (6) yields an equation for perturbation vertical velocity at the top of boundary layer:

$$
w^{\prime}=B_{1} v^{\prime}+B_{2} \frac{\partial v^{\prime}}{\partial x}+B_{3} \frac{\partial^{2} v^{\prime}}{\partial x^{2}},
$$

where the coefficients consisting of basic-state quantities are

$$
\begin{aligned}
& B_{1}=\frac{2}{\bar{\zeta}_{a}}\left(\frac{\partial|\bar{v}|}{\partial x}-\frac{|\bar{v}|}{\bar{\zeta}_{a}} \frac{\partial \bar{\zeta}_{a}}{\partial x}\right), \\
& B_{2}=\frac{2|\bar{v}|}{\bar{\zeta}_{a}}-2 \operatorname{Ro} \frac{\bar{v}}{\bar{\zeta}_{a}^{2}}\left(\frac{\partial|\bar{v}|}{\partial x}-\frac{|\bar{v}|}{\bar{\zeta}_{a}} \frac{\partial \bar{\zeta}_{a}}{\partial x}\right), \quad \text { and } \\
& B_{3}=-\operatorname{Ro} \frac{|\bar{v}| \bar{v}}{\bar{\zeta}_{a}^{2}}
\end{aligned}
$$

In the classical theory, $B_{1}=B_{3}=0$ and $B_{2}=2|\bar{v}|$, because the variation of $\bar{v}$ is small compared to $f$, which results in $\bar{\zeta}_{a}=1$ as Ro $\rightarrow 0$. The terms appearing in the rhs of (8) in addition to that in the classic theory are due to the presence of basic-state flow in the boundary layer $\bar{u}_{b}$ and the spatial variability of $\bar{v}$. As a result, the coefficient $B_{2}$ defined by (10) is no longer positive definite and can possibly be negative. The hypothesis indicates that the instability occurs when $B_{2}<0$.

A series of numerical calculations for the equations for the free atmosphere [(3)-(5)] and vertical velocity $[(8)]$ have been performed to test the hypothesized instability by artificially setting the coefficients $B_{1}, B_{2}$, and $B_{3}$ (appendix C). It was confirmed that the perturbation of vertical velocity grows only when $B_{2}<0$, which is consistent with the proposed theory, and all the other cases such as $B_{1}<0$ or $B_{3}<0$ show decaying solutions.

\section{c. The condition for instability}

We here analytically derive the condition for the instability (i.e., $B_{2}<0$ ) in the 1.5 -layer system. With some algebra and using the fact that $|\bar{v}| \partial_{x} \bar{v}=\bar{v} \partial_{x}|\bar{v}|$ results in an alternative form of the condition

$$
\bar{v} \frac{\partial^{2} \bar{v}}{\partial x^{2}} \operatorname{Ro}^{2}+\frac{\partial \bar{v}}{\partial x} \operatorname{Ro}+1<0 .
$$

If we consider cases with $\bar{v}>0$, the most favorable case to satisfy the inequality (12) is when both the first- and second-order derivatives of velocity $\partial_{x} \bar{v}$ and $\partial_{x}^{2} \bar{v}$ are negative (see appendix B). In other words, the conditions correspond to when the relative vorticity is negative and the spatial gradient of vorticity is also negative. This flow field is expected to appear outside the peak of a jet (at larger $x$ than the peak velocity) where the velocity gradient is negative and the second-order derivative is also negative up to the inflection point. The unstable condition (12) can be rewritten as

$$
\text { Ror }>1 \text {, }
$$

where

$$
\Upsilon \equiv \frac{2 \bar{v} \partial_{x}^{2} \bar{v}}{-\partial_{x} \bar{v}-\left[\left(\partial_{x} \bar{v}\right)^{2}-4 \bar{v} \partial_{x}^{2} \bar{v}\right]^{1 / 2}}
$$

Thus, $\Upsilon$ is a function of the spatial distribution of $\bar{v}$ and the lhs of (13), or equivalently, the sign of $B_{2}$ depends on the velocity distribution as well as Ro. To satisfy the unstable condition (13), $\Upsilon$ must be positive, and both Ro and $Y$ should be large. Specifically, a larger magnitude of $v^{\prime}$ and $\partial_{x}^{2} \bar{v}$ and smaller magnitude of $\partial_{x} \bar{v}$ are more favorable. As expected above and will be shown later, these conditions are satisfied in a jetlike flow.

\section{d. Eigenvalue analysis}

We conducted numerical eigenvalue analyses for the 1.5-layer system to seek unstable modes produced by the proposed mechanism. The linearized equations for the free atmosphere [(3)-(5)] and those for the boundary layer [(6) and (7)] can be combined into a single equation for $v^{\prime}$ :

$$
\begin{aligned}
\left(D_{1}+D_{2} \frac{\partial}{\partial x}+D_{3} \frac{\partial^{2}}{\partial x^{2}}\right) \frac{\partial v^{\prime}}{\partial t}= & G_{1}\left(D_{4}+D_{5} \frac{\partial}{\partial x}\right. \\
& \left.+D_{6} \frac{\partial^{2}}{\partial x^{2}}+D_{7} \frac{\partial^{3}}{\partial x^{3}}\right) v^{\prime}
\end{aligned}
$$

where

$$
\begin{aligned}
& D_{1}=\frac{\mathrm{Fr}^{2}}{\mathrm{Ro}^{2}}+\frac{2}{\bar{\zeta}_{a}^{2}} \frac{\partial \bar{\zeta}_{a}}{\partial x} \frac{\partial \bar{h}}{\partial x}-2 \frac{\bar{h}}{\bar{\zeta}_{a}^{3}}\left(\frac{\partial \bar{\zeta}_{a}}{\partial x}\right)^{2}+\frac{\bar{h}}{\bar{\zeta}_{a}^{2}} \frac{\partial^{2} \bar{\zeta}_{a}}{\partial x^{2}}-\frac{1}{\bar{\zeta}_{a}} \frac{\partial^{2} \bar{h}}{\partial x^{2}}, \\
& D_{2}=-\frac{2}{\bar{\zeta}} \frac{\partial \bar{h}}{\partial x}+\frac{2 \bar{h}}{\bar{\zeta}_{a}^{2}} \frac{\partial \bar{\zeta}_{a}}{\partial x}, \\
& D_{3}=-\frac{\bar{h}}{\bar{\zeta}_{a}} \\
& D_{4}=\frac{2}{\zeta_{a}} \frac{\partial^{2}|\bar{v}|}{\partial x^{2}}-\frac{4}{\zeta_{a}^{2}} \frac{\partial|\bar{v}|}{\partial x} \frac{\partial \bar{\zeta}_{a}}{\partial x}+\frac{4|\bar{v}|}{\zeta_{a}^{3}}\left(\frac{\partial \bar{\zeta}_{a}}{\partial x}\right)^{2}-\frac{2|\bar{v}|}{\zeta_{a}^{2}} \frac{\partial^{2} \bar{\zeta}_{a}}{\partial x^{2}},
\end{aligned}
$$




$$
\begin{aligned}
D_{5}= & \frac{4}{\bar{\zeta}_{a}}\left(\frac{\partial|\bar{v}|}{\partial x}-\frac{|\bar{v}|}{\bar{\zeta}_{a}} \frac{\partial \bar{\zeta}_{a}}{\partial x}\right)-2 \operatorname{Ro} \frac{\bar{v}}{\bar{\zeta}_{a}}\left[\frac{1}{\zeta_{a}} \frac{\partial^{2}|\bar{v}|}{\partial x^{2}}-\frac{4}{\zeta_{a}^{2}} \frac{\partial|\bar{v}|}{\partial x} \frac{\partial \bar{\zeta}_{a}}{\partial x}\right. \\
& \left.-\frac{3|\bar{v}|}{\zeta_{a}^{3}}\left(\frac{\partial \bar{\zeta}}{\partial x}\right)^{2}-\frac{|\bar{v}|}{\zeta_{a}^{2}} \frac{\partial^{2} \bar{\zeta}_{a}}{\partial x^{2}}+\frac{1}{\bar{v}_{\bar{\zeta}}} \frac{\partial \bar{v}}{\partial x} \frac{\partial|\bar{v}|}{\partial x}\right], \\
D_{6}= & \frac{2|\bar{v}|}{\bar{\zeta}_{a}}-4 \operatorname{Ro} \frac{\bar{v}}{\bar{\zeta}_{a}^{2}}\left(\frac{\partial|\bar{v}|}{\partial x}-\frac{|\bar{v}|}{\bar{\zeta}_{a}} \frac{\partial \bar{\zeta}_{a}}{\partial x}\right), \text { and } \\
D_{7}= & -\operatorname{Ro} \frac{|\bar{v}| \bar{v}}{\bar{\zeta}_{a}^{2}} .
\end{aligned}
$$

In the classic theory in which the basic-state velocity is not strong and varies slowly in space, $D_{1}=0, D_{2}=0$, $D_{3}=-\bar{h}, D_{4}=0, D_{5}=0, D_{6}=2|\bar{v}|$, and $D_{7}=0$. The velocity perturbation that is a function of space $x$ and time $t$ is decomposed as $v^{\prime}(x, t)=\hat{v}(x) \exp (\sigma t)$, where $\sigma$ is the growth rate. When $\operatorname{Re}\{\sigma\}>0$, the disturbance grows exponentially. Substituting this into (15) yields the eigenvalue equation for the growth rate:

$$
\sigma \mathcal{L} \hat{\mathbf{v}}=\mathcal{L}_{b} \hat{\mathbf{v}}
$$

where $\mathcal{L}$ and $\mathcal{L}_{b}$ are matrices for the lhs and rhs of (15), respectively, and $\hat{\mathbf{v}}$ is a vector form of $\hat{v}$.

The eigenvalue equation [(16)] was discretized on a staggered grid system and eigenvalues and eigenvectors were computed with MATLAB. As in the test calculations in appendix $\mathrm{C}$, the spatial derivatives in (15) were discretized by the fourth-order central difference scheme, and periodic boundary conditions were used. We have tested the second-order difference scheme and found that the results are insensitive to the discretization scheme. The nondimensional domain width was 8 with 201 grid points and uniform grid spacing of 0.04 . The initial velocity field was a jetlike profile $\bar{v}(x)=$ $\exp \left[-\left(x_{c}-x\right)^{2} / \Delta_{L}^{2}\right]$ with a peak at $x_{c}=2$ and a spatialscale $\Delta_{L}=\Delta_{L}^{*} / L$, where $L$ is a length scale. The velocity was computed from $x=0$ to 4 , and then the value at $x=0$ \{i.e., $\left.\bar{v}(x)=\exp \left[-\left(x_{c}-x\right)^{2} / \Delta_{L}^{2}\right]-\exp \left(-x_{c}^{2} / \Delta_{L}^{2}\right)\right\}$ was subtracted to make the velocity zero at $x=0$. To satisfy the periodic boundary condition for $\bar{h}$, one other velocity profile that is symmetric to that from $x=0$ to 4 with the same peak magnitude and width but opposite sign was added from $x=$ to 8 ; that is, $\bar{v}(x \mid x \in[4,8])=$ $-\bar{v}(x \mid x \in[0,4])$ (Sugimoto et al. 2007). The basic-state depth $\bar{h}$ was in geostrophic balance with $\bar{v}$.

Figures $3 \mathrm{a}$ and $3 \mathrm{~b}$ show spatial profiles of $\bar{v}$, vertical absolute vorticity $\bar{\zeta}_{a}$, and Ror in (13) for a representative case, in which $\mathrm{Ro}=0.70, \mathrm{Fr}=0.10$, and $\Delta_{L}=0.74$, corresponding approximately to $V=20 \mathrm{~m} \mathrm{~s}^{-1}, f=5 \times 10^{-5} \mathrm{~s}^{-1}$, and $H=5 \times 10^{3} \mathrm{~m}$. The velocity has a peak value at $x=2$ and gradually decays in each side of peak, and the vorticity has positive and negative peaks at $x=1.52$ and
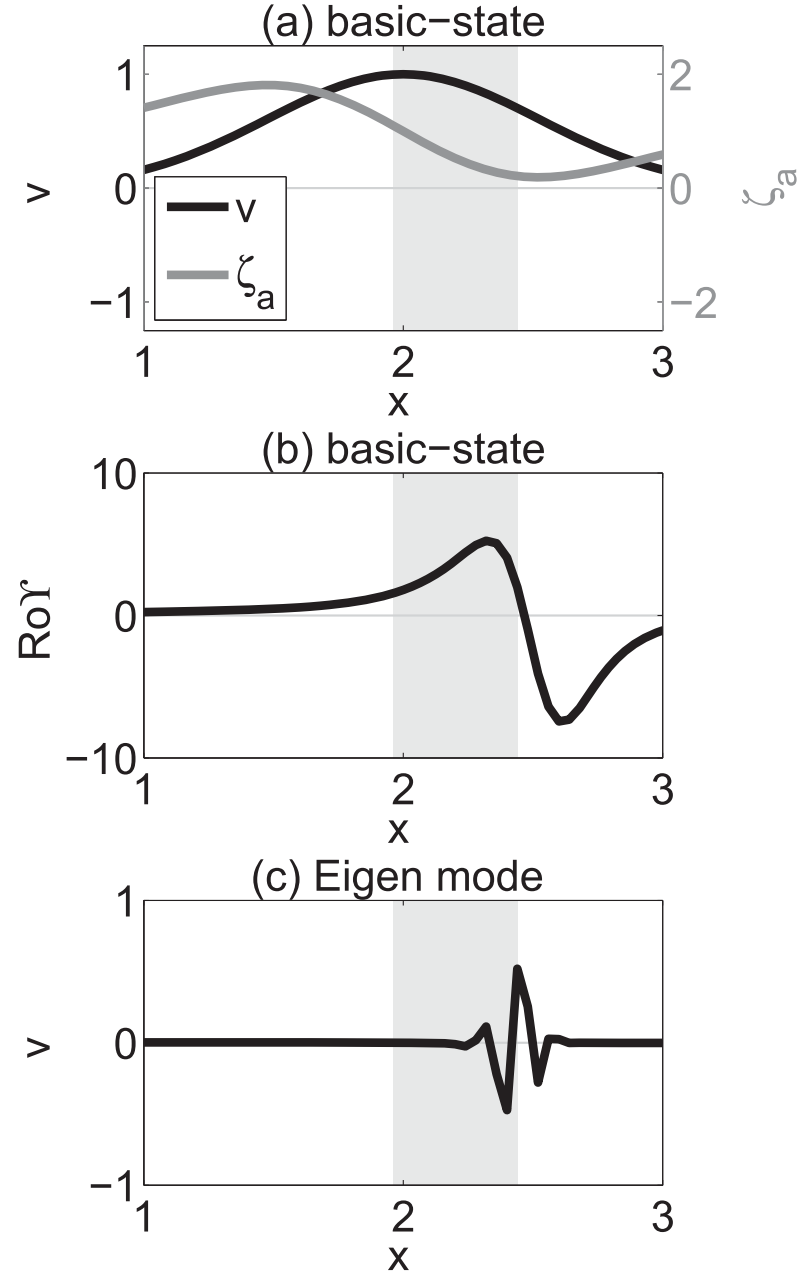

(d) Eigen mode

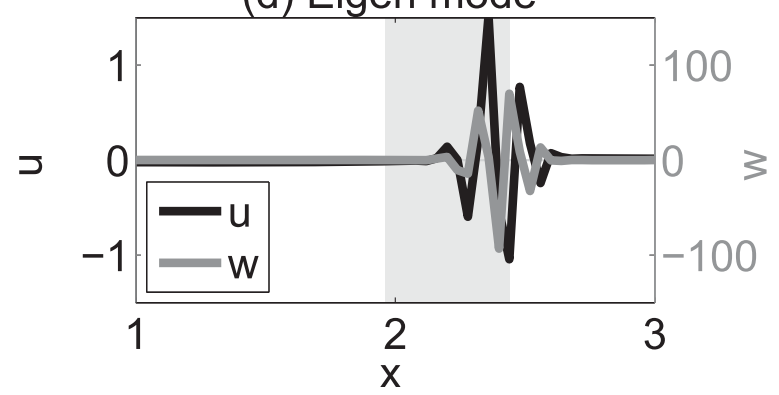

FIG. 3. Spatial profiles of (a) $\bar{v}$ (black line) and vertical absolute vorticity $\bar{\zeta}_{a}$ (gray line) and (b) $\Xi$ (black line) and Ror (gray line) in the control case with Ro $=0.70$ and $\Delta_{L}=0.74$ $\left(\operatorname{Ror}_{\max }=5.05\right)$. The eigenmodes of (c) $v^{\prime}$ and (d) $u^{\prime}$ (black line) and $w^{\prime}$ (gray line). The area where Ror $>1$ is highlighted by gray shading.

2.48. Ror increases with $x$ up to the maximum at $x=2.32$ and sharply decreases from there to $x=2.60$. The unstable condition, Ror $>1$ (13), is met in this case between $x=1.88$ and 2.36. As discussed in the previous section, the condition is more likely satisfied to the right 
TABLE 1. List of parameters in the eigenvalue analysis for Cartesian coordinates.

\begin{tabular}{clll}
\hline \hline Parameter & Min & Max & \multicolumn{1}{c}{ Description } \\
\hline Ro & 0.1 & 1.5 & Rossby number \\
$\Delta_{L}$ & 0.35 & 6.65 & Spatial scale of jetlike profile \\
$\Upsilon_{\max }$ & 0.08 & 4.24 & $\begin{array}{c}\text { Dimensionless quantity determining } \\
\text { unstable condition }\end{array}$ \\
\hline
\end{tabular}

of the peak of the jet where both the first-order and second-order derivatives of the basic-state velocity are negative. The range satisfying the unstable condition is highlighted in Fig. 3.

The eigenmodes with the maximum eigenvalue are shown in Figs. 3c and 3d. In this case, the real part of the maximum eigenvalue (i.e., growth rate) is 18.73 and corresponds to an $e$-folding time of $1.33 \times 10^{4} \mathrm{~s}(\approx 3.7 \mathrm{~h})$. The vertical velocity $w^{\prime}$ was calculated using (8) from the eigenmode of $v^{\prime}$, and then $u^{\prime}$ was diagnosed from (3) to (5). The plot of $v^{\prime}$ has a sharp positive peak at $x=2.36$. The eigenvector of $w^{\prime}$ also has sharp peaks around the $v^{\prime}$ peak. Finally, $u^{\prime}$ is largely negative at the positive peak of $v^{\prime}$ and positive at the negative peak. These profiles of velocities are basically consistent with the proposed theory.

We conducted a series of eigenvalue analyses for wide ranges of Ro and the spatial parameter $\Delta_{L}$ that controls the width of the velocity profile and hence changes the spatial derivatives of $\bar{v}$. Specifically, $\Upsilon$ monotonically increases with $\Delta_{L}$. According to the theoretical condition for the instability in (13), Ro and $\Delta_{L}$ are the key parameters to determine the stability. The parametersweep ranges are listed in Table 1 . Meanwhile, Fr and $G_{1}$ were fixed as 0.1 and 1.0, respectively, in all the calculations, because they do not affect the stability criteria.

Figure 4 displays a phase diagram in space of two parameters: Ro and the domain maximum of $\Upsilon$. The neutral curve, $\operatorname{Ror}=1$, is shown by the black line. The circles indicate experiments in which the real part of the maximum eigenvalue (growth rate) is positive and the domain-maximum $w^{\prime}$ is greater than 1.0. The $\times$ marks indicate experiments that do not satisfy both the criteria. Note that the gray circles represent experiments in which the real part of the eigenvalue is positive but the minimum value of basic-state absolute vorticity in the domain is negative, which is generally unphysical. The eigenvalue analyses show that the vertical velocity amplifies when the theoretical condition (13) is met. The stable and unstable regimes are clearly separated by the neutral curve Ror $=1$. Beyond the neutral curve, the solutions are mostly unstable, and growing modes are obtained. It should be noted that the negative absolute vorticity regime also appears beyond the neutral

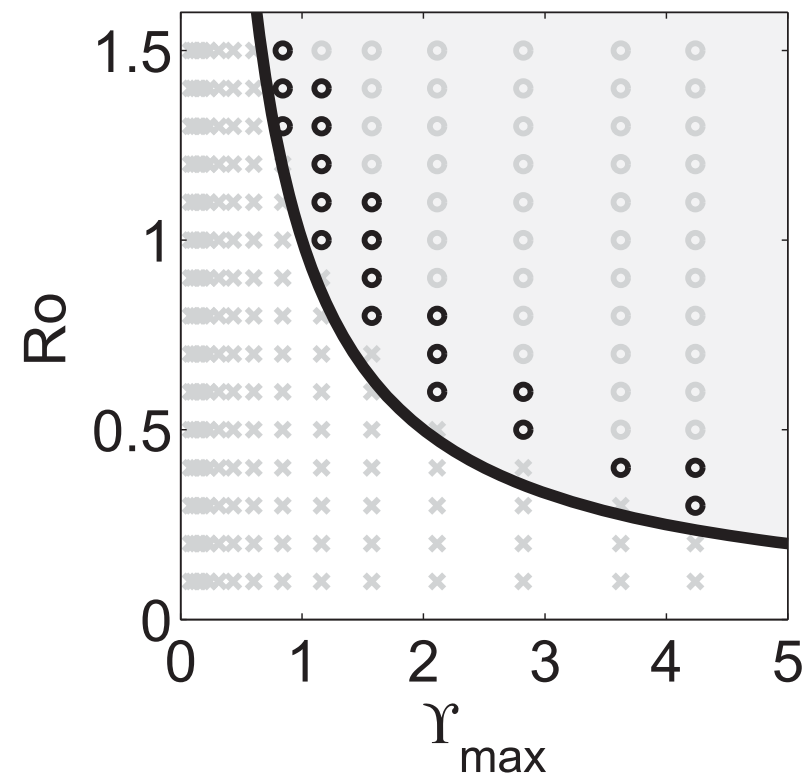

FIG. 4. Phase diagram in the parameter space (domain maximum of $\Upsilon$ and Ro) in the series of eigenvalue analyses. The black circles represent the experiments in which the real part of maximum growth rate is positive and maximum value of $w^{\prime}$ is greater than 1.0, whereas the gray circles indicate those in which the domain minimum of basic-state absolute vorticity is negative. The $\times$ marks are the experiments that do not meet the criteria. The line stands for the neutral curve in (13), Ror $=1$.

curve. The unstable regime with the minimum vorticity greater than 0 (black circles) is sandwiched in a narrow range between the neutral curve and the negative vorticity regime. It is indicated that the instability will occur when the unstable condition is satisfied but under limited environmental conditions.

\section{e. Numerical integrations of nonlinear equation set}

A series of numerical integrations for the nondimensional form of the nonlinear equation set (A7)(A11) were performed with variations of the two parameters, Ro and $\mathrm{r}$. We added a tendency term to the momentum equation for the $x$-direction in the free atmosphere (A8) to allow ageostrophic components. The dimensionless form of $u$ equation used here is

$$
G_{2} \frac{\partial u}{\partial t}=-\frac{\operatorname{Ro}}{\operatorname{Fr}^{2}} \frac{\partial h}{\partial x}+v
$$

where $G_{2}=\left(C_{D} V\right)^{2} /(f H)^{2}=\left(\operatorname{Ro} C_{D} L / H\right)^{2}$ and is determined by using the parameter values shown in Table 2 . The mass conservation and $y$-momentum equations in the boundary layer [(A10) and (A11)] were combined into an equation for $w$.

The equations were solved numerically using a finite difference method. As in the eigenvalue analysis, the 
TABLE 2. List of parameters that are constant in this study.

\begin{tabular}{cccl}
\hline \hline Parameter & Value & Unit & \multicolumn{1}{c}{ Description } \\
\hline $\mathrm{Fr}$ & 0.1 & - & Froude number \\
$G_{1}$ & 1.0 & - & Dimensionless quantity \\
$C_{D}$ & $1.5 \times 10^{-3}$ & - & Drag coefficient \\
$g^{*}$ & 9.8 & $\mathrm{~m} \mathrm{~s}^{-2}$ & Gravitational acceleration \\
$L$ & $1000.0 \times 10^{3}$ & $\mathrm{~m}$ & Scale of horizontal length \\
$H_{b}$ & $1.0 \times 10^{3}$ & $\mathrm{~m}$ & Scale of boundary layer depth \\
\hline
\end{tabular}

quantities were allocated on a staggered grid system. The time and space derivatives in the equations were discretized by the third-order Runge-Kutta method and by the fourth-order central difference scheme, respectively. At the first time step, $w$ was estimated from $v$, and then the equations for the free atmosphere were stepped forward. The integration was conducted up to $t=0.5$, dimensionally equivalent to 2.3 days, with a time step of $\Delta t=0.001$. The domain size, grid spacing, and boundary condition were the same as in the eigenvalue analysis. The profile of $v$ used in the eigenvalue analysis (Fig. 3a) was also applied to the initial field, which was balanced with the fluid depth. The experimental parameters and their range were also identical to the eigenvalue analysis.

Figure 5 shows spatial distributions of velocities at $t=0.02$ in the control case, in which Ro $=0.80$ and $\Delta_{L}=0.74$. The vertical velocity has a positive peak at $x=2.40$, which is the same location as the eigenvalue analysis (cf. Fig. 3c) and a negative peak near the positive one. There is a sharp peak in the $u$ distribution around the positive peak of $w$, whereas $u$ is negative at the both sides of the peak. The negative $u$ transports absolute vertical vorticity around the peak of $v$ where the vorticity is not weak compared with the negative $u$ region outside of the peak. As a result, $v$ is amplified around the peak of initial distribution. The velocity distributions in the nonlinear integration are qualitatively consistent with the eigenvalue analysis and the present hypothesis.

Figure 6a displays a space-time cross section of $v$ in the control case and time series of domain-maximum $w$. The maximum $w$ amplifies with time. The maximum $w$ keeps growing until the end of the simulation, and the locations of velocity peaks seen in Fig. 5 are nearly fixed in space. The spatial profiles of velocity during the integration (Fig. 5) strongly suggest that the proposed feedback works to amplify $w$. Since no explicit diffusion is incorporated into the discretized equation set, the linearly unstable modes continue to grow.

Figure 7 depicts a phase diagram in Ro and the domain-maximum $r$. The experiments in which the domainmaximum $w$ amplifies and exceeds $5 \mathrm{~m} \mathrm{~s}^{-1}$ are observed

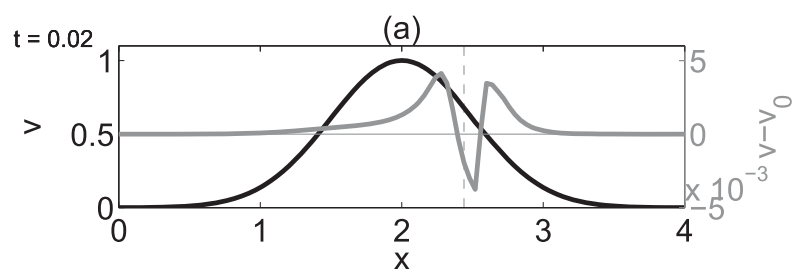

(b)
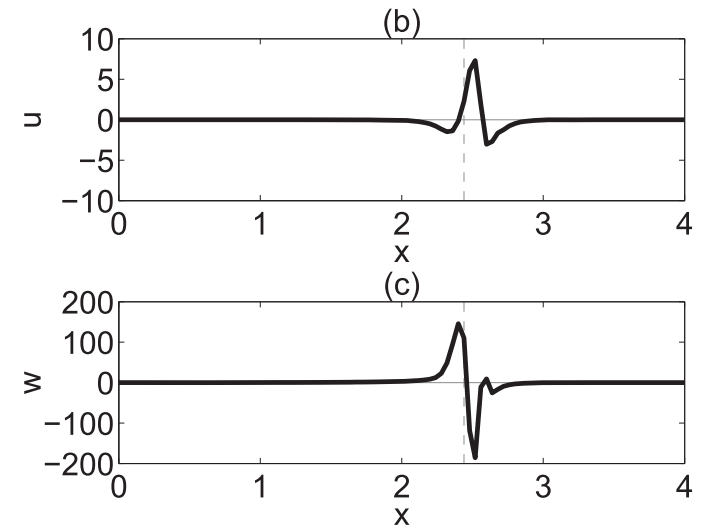

FIG. 5. Spatial profiles of (a) $v$ (black) and its deviation from the initial value $\left(v-v_{0}\right)$ (gray), (b) $u$, and (c) $w$ at $t=0.02$ in the control case of numerical integrations of nonlinear equations, in which $\mathrm{Ro}=$ 0.80 and $\Delta_{L}=0.71$. The vertical dashed lines indicate the location of maximum $w$.

in the unstable regime derived from the linear theory, Ror $>1$. The amplifying regime is clearly separated by the neutral curve from the regime in which the maximum $v$ decays after the initiation of integration $(\times$ marks in the figure). The theoretically derived unstable regime also includes a regime in which the domain-minimum absolute vertical vorticity is negative at the initial time. These features are quite similar to those obtained in the eigenvalue analyses (cf. Fig. 4). It should be noted that unlike the eigenvalue analyses, $w$ does not amplify in several cases close to the neutral curve. In these cases, the maximum $w$ decays rather than amplifies. The failure of unstable modes to grow when the parameters are close to the neutral curve may be due to nonlinear terms.

\section{Theory in cylindrical coordinates}

We here extend the theory developed in section 2 to cylindrical coordinates. The dimensionless governing equations are obtained in appendix D. The linearized mass conservation and radial and tangential momentum equations for free atmosphere are given as

$$
\begin{aligned}
\frac{1}{\operatorname{Ro} G_{1}} \frac{\partial h^{\prime}}{\partial t} & =-\frac{1}{r} \frac{\partial r u^{\prime} \bar{h}}{\partial r}+w^{\prime}, \\
\frac{\operatorname{Ro}}{\operatorname{Fr}^{2}} \frac{\partial h^{\prime}}{\partial r} & =v^{\prime} \bar{\xi}
\end{aligned}
$$



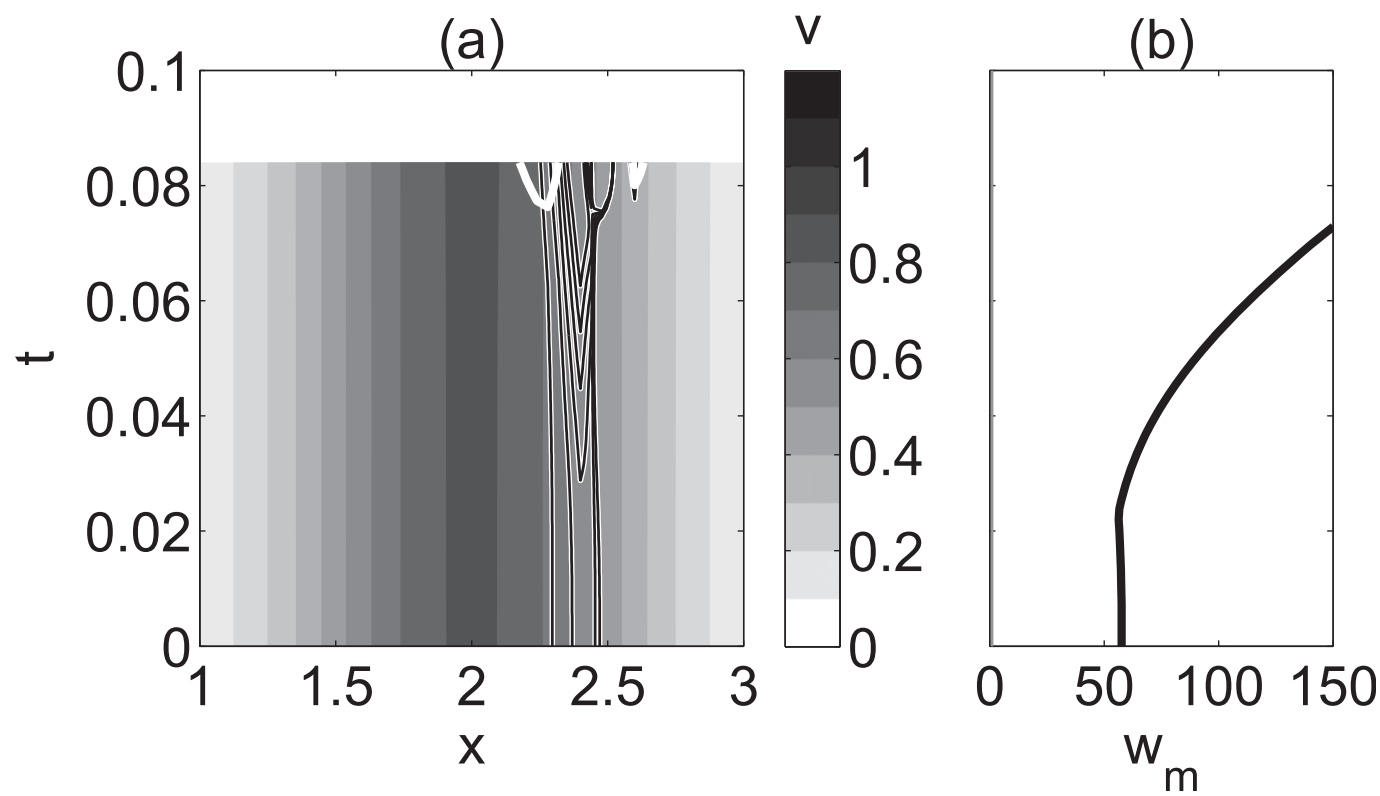

FIG. 6. (a) Time-space cross section of $v$ (shaded) and $w$ (black contour) in the control case. The contour for the vertical velocity is drawn from 0 to 50 at maximum with an interval of 10 . The white contour indicates where $v$ is 0.1 greater than the initial value. (b) Time series of domain-maximum $w$.

$$
\frac{1}{G_{1}} \frac{\partial v^{\prime}}{\partial t}=-u^{\prime} \overline{\zeta_{a}}
$$

and linearized equations for boundary layer are

$$
\begin{gathered}
-\frac{1}{r} \frac{\partial r u_{b}^{\prime} h_{b}}{\partial r}=w^{\prime} \\
u_{b}^{\prime} \bar{\zeta}_{a}+\bar{u}_{b} \operatorname{Ro}\left(\frac{\partial v^{\prime}}{\partial r}+\frac{v^{\prime}}{r}\right)=-\frac{2|v|}{h_{b}} v^{\prime}
\end{gathered}
$$

where $\bar{\xi}=\operatorname{Ro} 2 \bar{v} / r+1$ and $\bar{\zeta}_{a}=\operatorname{Ro}\left(\partial_{r} \bar{v}+\bar{v} / r\right)+1$ is basicstate absolute vertical vorticity. The basic-state radial velocity in the boundary layer $\bar{u}_{b}$ is given by the azimuthal momentum equation [(D12)]:

$$
\bar{u}_{b}=-\frac{|\bar{v}| \bar{v}}{h_{b} \bar{\zeta}_{a}}
$$

The perturbation vertical velocity at the top of boundary layer is obtained by combining (21) and (22) with the use of (23):

$$
w^{\prime}=2 \hat{\Xi}\left(E_{1} v^{\prime}+E_{2} r \frac{\partial v^{\prime}}{\partial r}+E_{3} r^{2} \frac{\partial^{2} v^{\prime}}{\partial r^{2}}\right),
$$

where

$$
E_{1}=2-\operatorname{Ro}\left(r \frac{\partial \Xi}{\partial r}+\Xi\right)+\frac{r}{\Xi} \frac{\partial \Xi}{\partial r},
$$

$$
\begin{aligned}
& E_{2}=1-\operatorname{Ro}\left(r \frac{\partial \Xi}{\partial r}+2 \Xi\right), \quad \text { and } \\
& E_{3}=-\operatorname{Ro} \frac{\Xi}{2},
\end{aligned}
$$

and

$$
\begin{aligned}
& \Xi=\frac{\bar{\Omega}}{\bar{\zeta}_{a}}, \\
& \hat{\Xi}=\frac{|\bar{\Omega}|}{\bar{\zeta}_{a}},
\end{aligned}
$$

are the ratio of angular velocity $\bar{\Omega}=\bar{v} / r$ to absolute vorticity. When the wind speed is not so fast in the free atmosphere that the advection term by the basicstate velocity is negligible and $v$ does not vary in space, $E_{1}=2, E_{2}=1, E_{3}=0, \Xi=\bar{\Omega}, \hat{\Xi}=|\bar{\Omega}|$, and $w^{\prime}=$ $2|\bar{v}|\left(2 v^{\prime} / r+\partial_{r} v^{\prime}\right)$. This converges to the classic theory where $w^{\prime}$ is proportional to the perturbation vorticity.

The necessary condition for the proposed instability is $E_{2}<0$. Using (23), this condition can be written as

$$
\operatorname{Ro}\left(r \frac{\partial \Xi}{\partial r}+2 \Xi\right)>1
$$

The condition is more likely to be satisfied as the factor in parentheses increases. Since we only consider cases in which $\bar{v}>0$ and $\bar{\zeta}_{a}>0$, and hence $\Xi>0$, the second term 


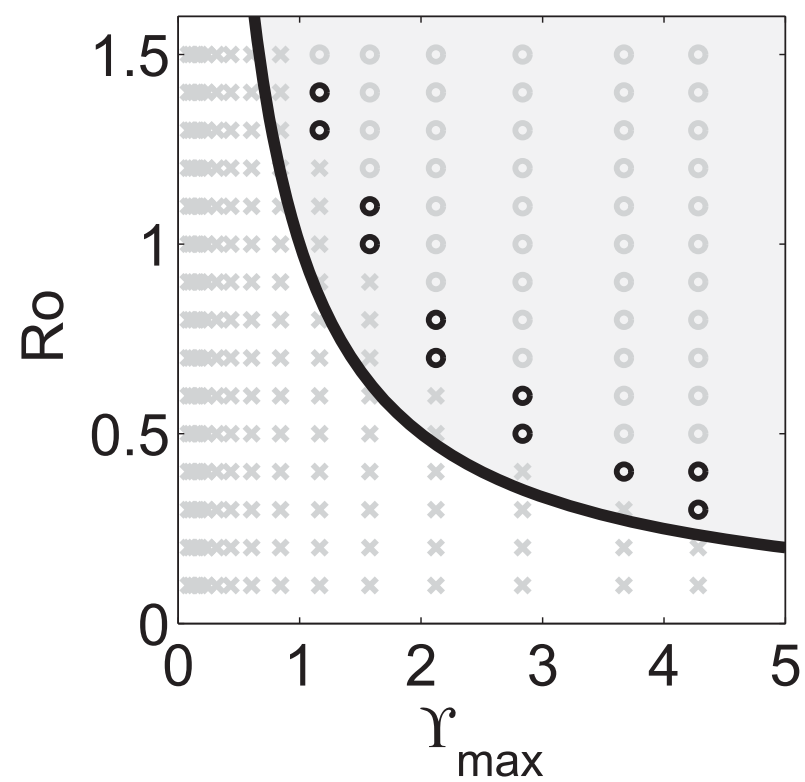

FIG. 7. As in Fig. 4, but for the results of nonlinear integrations. The circles indicate experiments in which the peak $w$ exceeds 5.0 during the integration period. The gray circles represent experiments in which the minimum absolute vorticity in the domain is negative at the initial time. The gray $\times$ marks show the experiments in which velocities decay as in the classic Ekman theory.

in the parentheses is always positive, whereas the first term can be either positive or negative. Thus, the terms in the parentheses are positive once $\partial_{r} \Xi$ is larger than 0 , which can be written as

$$
\frac{\partial \Xi}{\partial r}=\frac{1}{\bar{\zeta}_{a}} \frac{\partial \bar{\Omega}}{\partial r}-\frac{\bar{\Omega}}{\bar{\zeta}_{a}^{2}} \frac{\partial \bar{\zeta}_{a}}{\partial r}>0
$$

Since the angular velocity is larger than 0 , it is rewritten using (28) as

$$
\frac{\partial \bar{\zeta}_{a}}{\partial r}<\frac{\bar{\zeta}_{a}}{\bar{\Omega}} \frac{\partial \bar{\Omega}}{\partial r}=\frac{1}{\Xi} \frac{\partial \bar{\Omega}}{\partial r}
$$

It indicates that, for the instability, the radial gradient of vorticity should be more negative than the right-hand side, because $\partial_{r} \bar{\Omega}=\left(\partial_{r} \bar{v}-\bar{v} / r\right) / r$ rarely takes positive values outside the RMW. Furthermore, it is more favorable if the absolute vorticity is small, angular velocity is large, magnitude of radial gradient of angular velocity $\left|\partial_{r} \bar{\Omega}\right|$ is small, and magnitude of radial gradient of absolute vorticity $\left|\partial_{r} \bar{\zeta}_{a}\right|$ is large. The importance of small absolute vorticity and large negative gradient of vorticity is consistent with Kepert (2013).

Equation (24) for $w^{\prime}$ is a function of $\bar{v}$, and hence, the rhs of condition (30) is determined by the radial profile of $\bar{v}$ and Ro. To separate Ro and terms associated with $\bar{v}$, the condition, $E_{2}<0$, is written as

$$
\text { Ror }>1 \text {, }
$$

where, in a vortex,

$$
\mathrm{Y} \equiv \frac{-4\left(\partial_{r} \bar{v}\right)^{2}+2 \bar{v} \partial_{r}^{2} \bar{v}-2(\bar{v} / r) \partial_{r} \bar{v}-2(\bar{v} / r)^{2}}{\left(3 \partial_{r} \bar{v}+\bar{v} / r\right)+\left[\left(\partial_{r} \bar{v}\right)^{2}+4 \bar{v} \partial_{r}^{2} \bar{v}+2(\bar{v} / r) \partial_{r} \bar{v}-3(\bar{v} / r)^{2}\right]^{1 / 2}}
$$

The value of $\Upsilon$ tends to increase with the larger angular velocity $\bar{v} / r$ and absolute value of second-order derivative of velocity $\left|\partial_{r}^{2} v\right|$ and smaller first-order derivative of velocity $\left|\partial_{r} v\right|$, which is similar to the result obtained above.

\section{Eigenvalue analysis in a cylindrical coordinate}

To investigate the stability of the linear system developed in the previous section, linear stability analyses are conducted by solving an eigenmode equation for $v^{\prime}$. The equations for the free atmosphere [(18)-(20)] and the equation for vertical velocity [(24)] are combined into a single equation for $v^{\prime}$ :

$$
\begin{aligned}
& \left(F_{1}+F_{2} \frac{\partial}{\partial r}+F_{3} \frac{\partial^{2}}{\partial r^{2}}\right) \frac{\partial v^{\prime}}{\partial t} \\
& \quad=G_{1}\left(F_{4}+F_{5} r \frac{\partial}{\partial r}+F_{6} r^{2} \frac{\partial^{2}}{\partial r^{2}}+F_{7} r^{3} \frac{\partial^{3}}{\partial r^{3}}\right) v^{\prime}
\end{aligned}
$$

where

$$
\begin{aligned}
F_{1}= & \frac{\operatorname{Fr}^{2}}{\operatorname{Ro}^{2}} \bar{\xi}+\frac{\bar{h}}{\bar{\zeta}_{a}^{2}} \frac{\partial^{2} \bar{\zeta}_{a}}{\partial r^{2}}-\frac{2 \bar{h}}{\bar{\zeta}_{a}^{3}}\left(\frac{\partial \bar{\zeta}_{a}}{\partial r}\right)^{2} \\
& +\left(2 \frac{\partial \bar{h}}{\partial r}+\frac{\bar{h}}{r}\right) \frac{1}{\bar{\zeta}_{a}^{2}} \frac{\partial \bar{\zeta}_{a}}{\partial r}-\frac{1}{\bar{\zeta}_{a}} \frac{\partial}{\partial r}\left(\frac{1}{r} \frac{\partial r \bar{h}}{\partial r}\right), \\
F_{2}= & \frac{2 \bar{h}}{\bar{\zeta}_{a}^{2}} \frac{\partial \bar{\zeta}_{a}}{\partial r}-\frac{1}{\bar{\zeta}_{a}}\left(2 \frac{\partial \bar{h}}{\partial r}+\frac{\bar{h}}{r}\right), \\
F_{3}= & -\frac{\bar{h}}{\bar{\zeta}}, \\
F_{4}= & 6 \frac{\partial \hat{\Xi}}{\partial r}+2 r \frac{\partial^{2} \hat{\Xi}}{\partial r^{2}}-2 \operatorname{Ro}\left[3 \Xi \frac{\partial \Xi}{\partial r}+r\left(\frac{\partial \Xi}{\partial r}\right)^{2}+r \frac{\partial^{2} \Xi}{\partial r^{2}}\right],
\end{aligned}
$$




$$
\begin{aligned}
F_{5}= & 4 \frac{\partial \hat{\Xi}}{\partial r}+\frac{6 \hat{\Xi}}{r}-2 \operatorname{Ro}\left[7 \Xi \frac{\partial \Xi}{\partial r}+r\left(\frac{\partial \Xi}{\partial r}\right)^{2}\right. \\
& \left.+r \frac{\partial^{2} \Xi}{\partial r^{2}}+\frac{3 \Xi^{2}}{r}\right], \\
F_{6}= & \frac{2 \hat{\Xi}}{r}-2 \operatorname{Ro}\left[2 \Xi \frac{\partial \Xi}{\partial r}+\frac{3 \Xi^{2}}{r}\right], \\
F_{7}= & \operatorname{Ro}\left(-\frac{\Xi^{2}}{r}\right) .
\end{aligned}
$$

Substituting a waveform solution, $v^{\prime}=\hat{v}(r) \exp (\sigma t)$, where $\sigma$ is the growth rate, into (35) yields the following eigenvalue equation:

$$
\sigma \mathcal{L} \hat{\mathbf{v}}=\mathcal{L}_{b} \hat{\mathbf{v}}
$$

where $\mathcal{L}$ and $\mathcal{L}_{b}$ are the matrices in the lhs and rhs of (35) and $\hat{\mathbf{v}}$ is the vector form of $\hat{v}$.

The basic-state quantities were produced from a radial profile of vorticity in Kepert (2013) that used a smoothing function from Willoughby et al. (2006):

$$
\begin{aligned}
\zeta_{a}^{*}\left(r^{*}\right)= & \mathcal{W}_{1}\left(r^{*}\right) \zeta_{a, \mathrm{~A}}^{*}+\left[\mathcal{W}_{2}\left(r^{*}\right)-\mathcal{W}_{1}\left(r^{*}\right)\right] \zeta_{a, \mathrm{~B}}^{*} \\
& +\left[1-\mathcal{W}_{2}\left(r^{*}\right)\right] \zeta_{a, \mathrm{C}}^{*}\left(\frac{r^{*}}{r_{2}^{*}}\right)^{-1-\alpha}
\end{aligned}
$$

where $\mathcal{W}$ is a weight function (Willoughby et al. 2006) and is the same as in Kepert (2013). The filter width $L_{b}$ was $15 \mathrm{~km}$. The vorticities in the regions $\mathrm{A}, \mathrm{B}$, and $\mathrm{C}$ are

$$
\zeta_{a}^{*}= \begin{cases}\zeta_{a, \mathrm{~A}}^{*}=\zeta_{a 0}^{*} & r^{*} \leq r_{m}^{*} \\ \zeta_{a, \mathrm{~B}}^{*}=C_{\mathrm{A}} \zeta_{a 0}^{*} & r_{m}^{*}<r^{*} \leq D_{L} r_{m}^{*} \\ \zeta_{a, \mathrm{C}}^{*}=C_{\mathrm{A}} C_{\mathrm{B}} \zeta_{a 0}^{*}\left(\frac{r^{*}}{D_{L} r_{m}^{*}}\right)^{-1-\alpha} & r^{*}<D_{L} r_{m}^{*}\end{cases}
$$

In the analyses, $\zeta_{a 0}^{*}$ was given by $\operatorname{Ro} f^{*}$, where $f^{*}$ was fixed as $5 \times 10^{-5} \mathrm{~s}^{-1}$. A radial profile of dimensional tangential velocity $v^{*}$ was obtained by radial integration of (37). Then $v^{*}$ was normalized by the maximum value. Fluid depth and radial velocity in the boundary layer were obtained by gradient wind balance and (23).

The radial dimension was discretized on a staggered grid system with uniform grid spacing of 0.04 . The domain size was 80.0 (i.e., 80 times the RMW) with 2001 grid points. The radial derivatives were discretized by a fourth-order central difference scheme. At the boundaries $(r=0$ and 80$), v^{\prime}=0$.
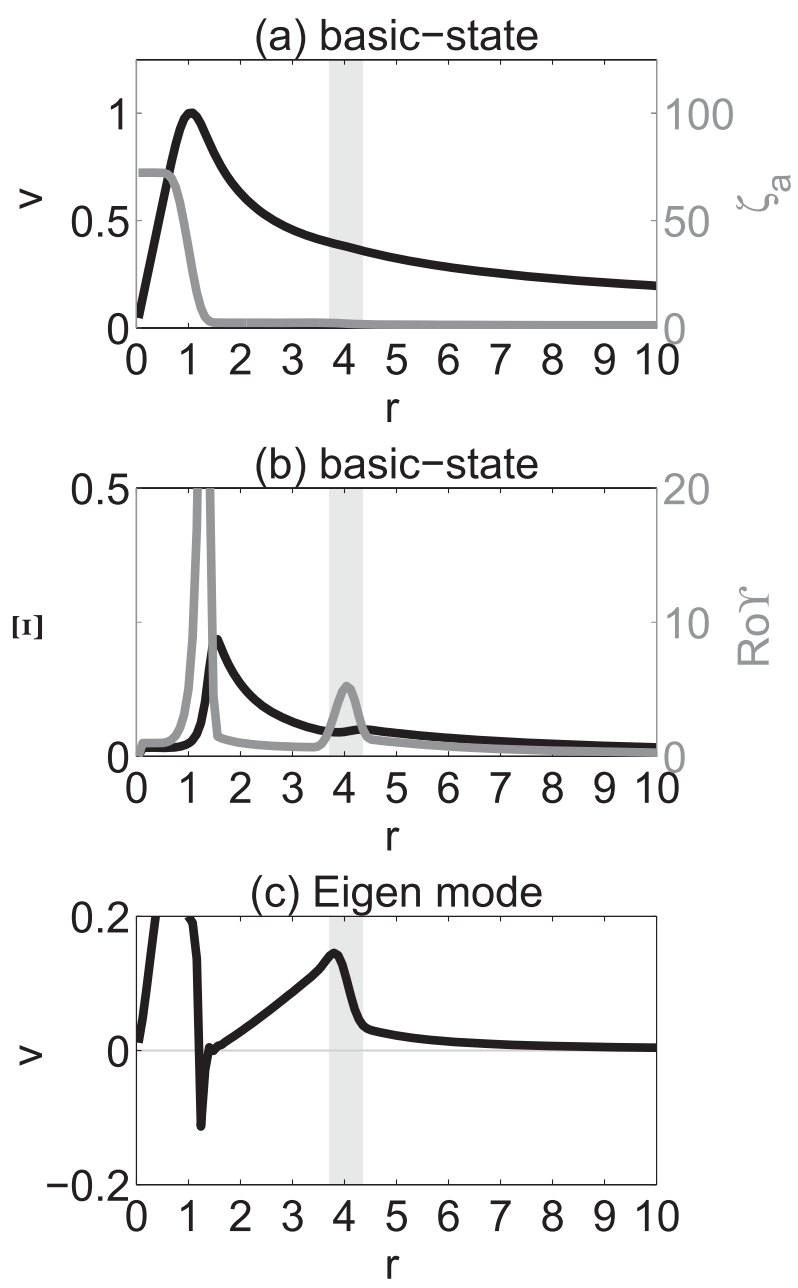

(d) Eigen mode

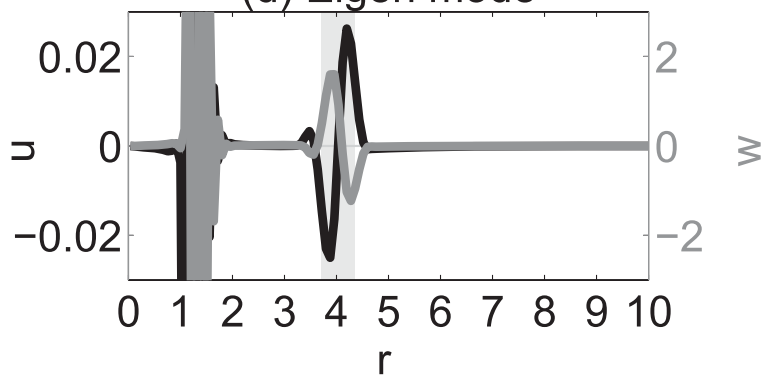

FIG. 8. Radial profiles of basic-state (a) tangential velocity $\bar{v}$ (black line) and absolute vorticity $\bar{\zeta}_{a}$ (gray line) and (b) $\Xi$ (black) and Ror (gray) in the control case $\left(\mathrm{Ro}=50, \mathrm{D}=4.0, C_{\mathrm{A}}=0.03\right.$, $C_{\mathrm{B}}=0.5, \alpha=0.8$ ). Radial profiles of eigenmodes of (c) $v^{\prime}$, (d) $u^{\prime}$ (black), and $w^{\prime}$ (gray).

Figures $8 \mathrm{a}$ and $8 \mathrm{~b}$ show radial profiles of basic-state $v, \zeta_{a}, \Xi$ and Ror in the control case ( $\mathrm{Ro}=50, D_{L}=4.0$, $\left.C_{\mathrm{A}}=0.02, C_{\mathrm{B}}=0.5, \alpha=0.5\right) ; \bar{v}$ has a peak at $r=1.0$ and decays monotonically outside the RMW. The vorticity is constant inside the RMW and rapidly decreases around the RMW. There is a small local decrease of vorticity at 
TABLE 3. List of parameters in the eigenvalue analysis for cylindrical coordinates.

\begin{tabular}{llcll}
\hline \hline Parameter & Min & Max & Increment & \\
\hline Ro & 20.0 & 87.5 & 3.75 & Description \\
$\delta$ & 1.0 & 5.5 & 0.25 & Rossby number \\
$C_{\mathrm{A}}$ & 0.005 & 0.055 & 0.0025 & Vodial distance of secondary vorticity jump \\
$C_{\mathrm{B}}$ & 0.5 & 1.0 & 0.025 & Vorticity ratio inside/outside the RMW \\
$\alpha$ & 0.1 & 1.0 & 0.05 & Radial decay of vorticity outside the secondary jump \\
$\Upsilon_{\max }$ & 0.08 & 4.24 & - & Dimensionless quantity determining unstable condition \\
\hline
\end{tabular}

$r=4.0$. However, this jump is more than 10 times smaller than that in Kepert (2013). Hence, unlike most of the cases in Kepert (2013), $\bar{v}$ has no clear secondary peak around $r=4.0$. The ratio of angular velocity to absolute vorticity $\Xi$ has a peak outside the RMW and a small peak around $r=4.5$. Ror also has a sharp peak immediately outside the RMW and a peak around $r=4.0$, where the radial gradient of $\Xi$ is large. The unstable condition (33) is satisfied in $3.78 \leq r \leq 5.08$ in this case, which is highlighted in the panels.

The eigenmodes of $v^{\prime}, u^{\prime}$, and $w^{\prime}$ are shown in Figs. 8c and $8 \mathrm{~d}$. In the cylindrical coordinate model with the hurricane-like wind profile, Fig. $8 \mathrm{~b}$ also shows a large value of Ror around the RMW. Not surprisingly, a very unstable mode is also excited in that region. To isolate the instability in the SEF region, we select the unstable mode that has the fastest growth rate of $v^{\prime}$ but also has significant amplitude of $w^{\prime}$ at $r>1.5 \times \mathrm{RMW}$. The $u^{\prime}, v^{\prime}$, and $w^{\prime}$ profiles of this mode are shown in Figs. $8 \mathrm{c}$ and $8 \mathrm{~d}$; the instability around the RMW is also evident. The nondimensional growth rate is 52.4, and the corresponding dimensional $e$-folding time is $4.77 \times 10^{3} \mathrm{~s}(\sim 1.3 \mathrm{~h})$. The value of $v^{\prime}$ is large and positive around $r=4.0$ where Ror has a peak; $u^{\prime}$ is largely negative (positive) outside (inside) the radius of $r=4.0$, which is opposite to $w^{\prime}$ in phase.

We examine the sensitivity of the solution to Ro and vortex parameters $D_{L}, C_{\mathrm{A}}, C_{\mathrm{B}}$, and $\alpha$. Each parameter out of the five is changed to examine the sensitivity, while the other four are fixed as those in the control case (shown above). Table 3 shows the range of parameters. The minimum and maximum values of Ro are 20.0 and 87.5 , which approximately correspond to $v_{m}=25.0$ and $109.4 \mathrm{~m} \mathrm{~s}^{-1}$, provided that $r_{m}=25 \mathrm{~km}$ and $f_{0}=5 \times 10^{-5} \mathrm{~s}^{-1}$. The ratio of the radius of the second "vorticity jump" between the region B and C to the RMW $D_{L}$ varies from 2 to 11 . The coefficient controlling vorticity outside the RMW $C_{\mathrm{A}}$ is changed from 0.005 to 0.05 . The magnitude of secondary vorticity jump $C_{\mathrm{B}}$ is between 0.1 and 1.0. The radial decay parameter $\alpha$ is changed from 0.1 to 1.0.

Figure 9 shows the growth rate as a function of experimental parameters. The growth rate increases with Ro,
$D_{L}, C_{\mathrm{A}}$, and $\alpha$ and decreases with $C_{\mathrm{B}}$. Thus, the growth rate is larger with stronger TC intensity, smaller RMW, and lower Coriolis parameter. The secondary peak of velocity tends to form more outside. Interestingly, the growth rate is positive only between $r=2.5$ and 7.0: the disturbance cannot grow if it is too close to the RMW or too far from the RMW. The growth rate is large with large $C_{\mathrm{A}}$, corresponding to large vorticity and hence fast velocity outside the RMW. When the vorticity outside the RMW is not strong enough $\left(C_{\mathrm{A}}<0.02\right)$, unstable solutions are not obtained. Both the dependence on $C_{\mathrm{B}}$ and $\alpha$ indicate that the growth rate is larger when the radial vorticity gradient at the secondary vorticity jump is steeper. The large vorticity and fast velocity is consistent with the wind field discussion that has been discussed in the previous studies.

To summarize the series of parameter sensitivity experiments, the relationship between the growth rate and $r$ divided by $\Xi$ is shown in Fig. 10 . The growth rate monotonically increases with $\mathrm{r}(\mathrm{Ro} \Xi)^{-1}$. Whereas no analytical formula is obtained for $\sigma$, the figure indicates $\Upsilon(\operatorname{Ro} \Xi)^{-1}$ is a good scaling factor for the growth rate in the system; $\Upsilon(\operatorname{Ro} \Xi)^{-1}=r \Xi^{-1} \partial_{r} \Xi+2$, and the first term is written as

$$
\frac{r}{\Xi} \frac{\partial \Xi}{\partial r}=r\left(\frac{1}{\bar{\Omega}} \frac{\partial \bar{\Omega}}{\partial r}-\frac{1}{\bar{\zeta}_{a}} \frac{\partial \bar{\zeta}_{a}}{\partial r}\right) .
$$

Since $\bar{\Omega}$ and $\bar{\zeta}_{a}$ are positive and $\partial_{r} \bar{\Omega}$ is never positive, a large negative radial gradient of $\bar{\zeta}_{a}$ is required for positive $\Upsilon(\operatorname{Ro} \Xi)^{-1}$. Therefore, larger $\bar{\Omega}$, smaller $\bar{\zeta}_{a}$, smaller $\left|\partial_{r} \bar{\Omega}\right|$, and larger $-\partial_{r} \bar{\zeta}_{a}$ is more favorable. These are consistent with the analytically derived unstable condition in the previous section.

\section{SEF simulated in full-physics models}

We examine SEF simulated by two nonhydrostatic, full-physics numerical models. The unstable condition (33) was applied to the simulation results to test the hypothesis that the proposed theory accounts for the initial process of SEF. To calculate the unstable condition, (33) is dimensionalized as 
(a)

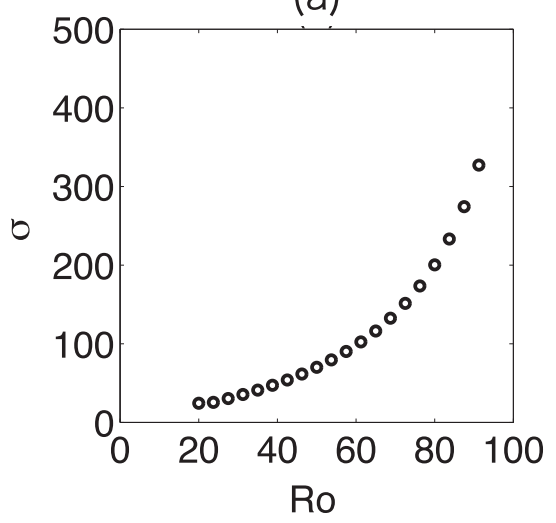

(c)

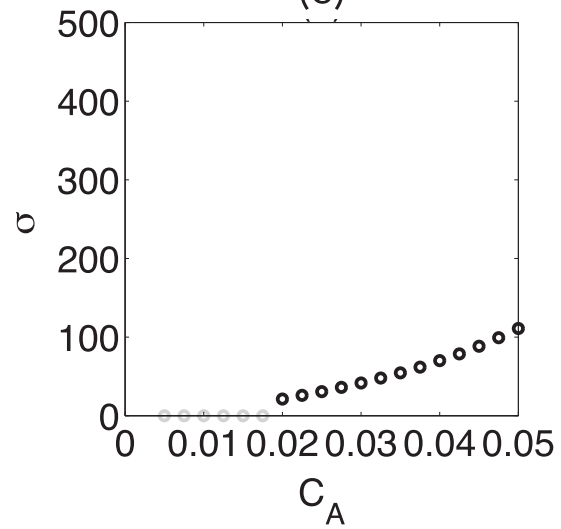

(b)

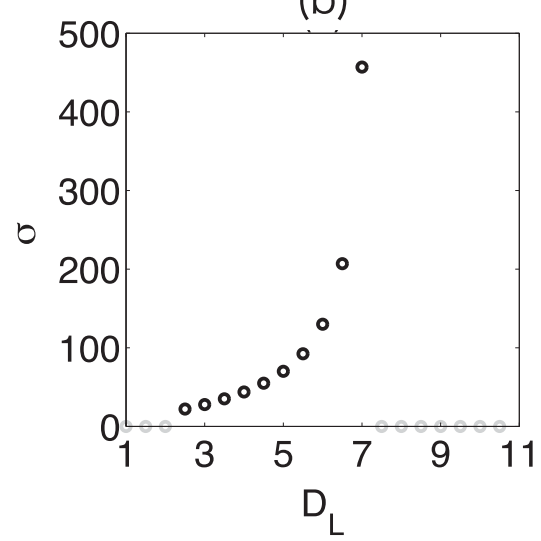

(d)

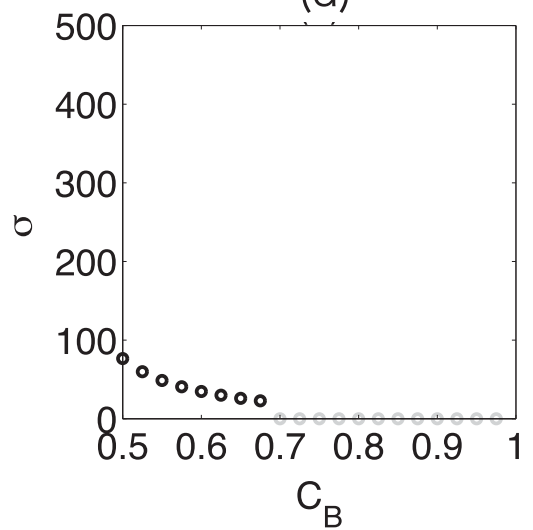

(e)

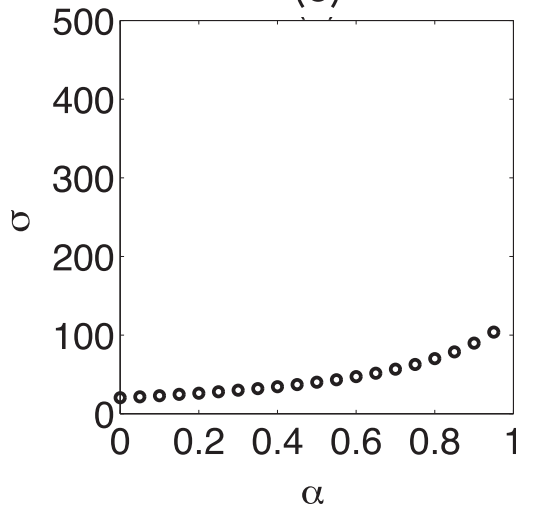

FIG. 9. Growth rate as a function of experimental parameters: (a) Ro; (b) radius of secondary jump of vorticity relative to the RMW, $D_{L}$; (c) ratio of vorticity in region $\mathrm{B}$ to inner core, $C_{\mathrm{A}}$; (d) ratio of vorticity in region $\mathrm{C}$ to $\mathrm{B}, C_{\mathrm{B}}$; and (e) vorticity decaying coefficient, $\alpha$. The black circles indicate unstable solutions $(\operatorname{Re}\{\sigma\}>0)$, whereas all stable or neutral solutions $(\operatorname{Re}\{\sigma\} \leq 0)$ indicated by gray circles are plotted at $\sigma=0$.

$$
\begin{aligned}
\Upsilon \equiv \frac{r^{*}}{\Xi^{*}} \frac{\partial \Xi^{*}}{\partial r^{*}}+2 \Xi^{*}= & \frac{\Omega^{*}}{\zeta_{a}^{*}}\left[\left(-\frac{1}{\zeta_{a}^{*}} \frac{\partial \zeta_{a}^{*}}{\partial r^{*}}\right.\right. \\
& \left.\left.+\frac{1}{\Omega^{*}} \frac{\partial \Omega^{*}}{\partial r^{*}}\right) r^{*}+2\right]>1 .
\end{aligned}
$$

Unlike the unstable condition for the linear model (33), Ro does not appear in the inequality as it is eliminated in the dimensionalization.

\section{a. Axisymmetric model}

A long-term simulation was performed using an axisymmetric version of Cloud Model 1 (CM1; version 17; Bryan and Fritsch 2002), which solves fully compressible, nonhydrostatic equations. The experimental setting was basically the same as Hakim (2013) in which a number of eyewall replacement cycle events were simulated during his long-term simulation for 500 days. The differences from Hakim's (2013) simulation are listed below. The initial mass, temperature, and water vapor mixing ratio was obtained from Jordan's (1958) tropical mean sounding for hurricane season. The Coriolis parameter was $5 \times 10^{-5} \mathrm{~s}^{-1}$, and the sea surface temperature was $301.15 \mathrm{~K}$, both of which were fixed during integration. The radiative effects were incorporated by the NASA Goddard scheme, which was called every $5 \mathrm{~min}$. The integration period was 60 days $(=1440 \mathrm{~h})$ with an output interval of $3 \mathrm{~h}$ for prognostic quantities. The grid spacing for the radial direction was $2 \mathrm{~km}$, whereas the spacing for the vertical direction was stretched from the bottom to top. The minimum grid spacing for the vertical direction was $0.25 \mathrm{~km}$ at the lowest model layer, stretching to $1.0 \mathrm{~km}$ near the top.

Figurea 11a and 11b show time series of TC intensity defined as the maximum tangential velocity at $z=2 \mathrm{~km}$ and its radius. The TC achieves maximum intensity around $t=340 \mathrm{~h}$ after an intensification phase, decreases to about $60 \mathrm{~m} \mathrm{~s}^{-1}$, and then is approximately steady afterward, which is qualitatively consistent with the longterm simulations in previous studies (Hakim 2011, 2013). We shall call the period after $t=500 \mathrm{~h}$ a quasi-steady state. During the quasi-steady state, rapid changes in 


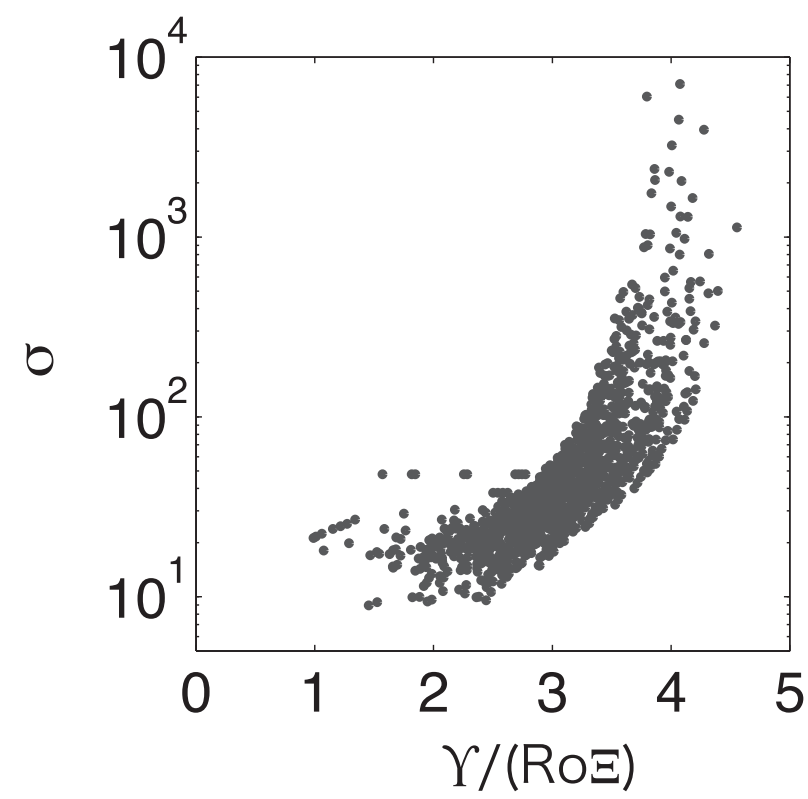

FIG. 10. Growth rate as a function of $\Upsilon /(\operatorname{Ro} \Xi)$ for a series of eigenvalue analyses. Each point indicates a member of analysis.

intensity and RMW are observed several times. As pointed out by Hakim (2011), the intensity oscillation appears to be due to the eyewall replacement cycle associated with SEF.

Figure $11 \mathrm{c}$ depicts a radius-height cross section of tangential and radial velocities and mixing ratio of hydrometeors averaged during the quasi-steady state. Both the velocity and cloud fields are consistent with the observational studies (e.g., Hawkins and Imbembo 1976): the tangential velocity has a peak at the top of the boundary layer $(\sim 1.5 \mathrm{~km})$ and decays in both the radial and vertical directions, the inflow and outflow regions appear at the surface and around the $15-\mathrm{km}$ level, and the hydrometeor mixing ratio is large around the RMW from the 1.5- to $15-\mathrm{km}$ altitudes.

Figure $11 \mathrm{~d}$ depicts a radius-time cross section of tangential velocity at $z=2 \mathrm{~km}$. The RMW is about $60 \mathrm{~km}$ during the quasi-steady state as seen in Fig. 11b. Interestingly, a strong velocity region periodically forms about every $80 \mathrm{~h}$, and the region proceeds inward after formation. The mixing ratio of hydrometeors and vorticity fields shows similar behavior to the tangential velocity (not shown). It is suggested that this cyclic process is the eyewall replacement cycle and formation of strong tangential velocity outside the RMW is SEF.

The SEF events were extracted from the simulation by applying the following conditions: the RMW is greater than that at the previous and next output time, the increase from the previous step is greater than $10 \mathrm{~km}$, and the new RMW is more than $20 \mathrm{~km}$ beyond its average value during the quasi-steady state $(\sim 60 \mathrm{~km}$ in this case). Applying the conditions, six SEF events were detected in the quasi-steady period, and the six events were combined to make composites.

Figure 12a displays a radius-time cross section of tangential velocity at $z=2 \mathrm{~km}$ subtracted from the temporal average from $t-t_{\mathrm{SEF}}=-40$ to $+40 \mathrm{~h}$ and $\mathrm{r}$, which are composited from the detected SEF events. The value of $\Upsilon$ is calculated from $v$ at $z=2 \mathrm{~km}$ at each output time after taking 1-2-1 average for $v$ for 20 times in the radial direction. A secondary peak of tangential velocity forms at $r=163 \mathrm{~km}$, which is about $100 \mathrm{~km}$ outside the RMW, $20 \mathrm{~h}$ before the time of SEF. The peak amplifies as it propagates inward. The $Y$ becomes significant $3 \mathrm{~h}$ before the secondary peak forms, and the unstable condition is satisfied; $\Upsilon$ is large around the RMW for the entire time frame, which is obviously due to the large radial gradient of vertical vorticity.

Figure $12 \mathrm{~b}$ shows the time series of $\Upsilon, \Xi$, and $\partial_{r} \zeta_{a}$, which are radially averaged from $r=135$ to $160 \mathrm{~km}$, where the secondary peak of tangential velocity forms; $\Upsilon$ clearly has a large value at $t-t_{\mathrm{SEF}}=-20 \mathrm{~h}$, which is immediately before the formation of the secondary peak. Since $\partial_{r} \zeta_{a}$ is large and negative at this time, the increase in $Y$ results from the enhanced radial vorticity gradient. It suggests that the proposed linear instability works to amplify the tangential velocity to form a secondary peak outside the RMW.

\section{b. Three-dimensional model}

We conducted a numerical simulation using a threedimensional full-physics numerical model, Weather Research and Forecasting (WRF) Model, version 3.4.1 (Skamarock et al. 2008). The experimental setting is described in appendix E.

Figures 13a and 13b show time series of TC intensity and RMW of the simulated TC. The TC experiences two different intensification phases and becomes quasi steady after $t=45 \mathrm{~h}$. The intensity weakens from $t=65$ to $76 \mathrm{~h}$ and reintensifies afterward. The RMW gradually decreases, jumps outward at $t=76 \mathrm{~h}$, and then decreases again. Structural evolution of the simulated TC (shown below) indicates that the sudden changes in TC intensity and RMW are associated with SEF and the eyewall replacement process. We define $t=76 \mathrm{~h}$ as the time of SEF.

Figure $13 \mathrm{c}$ depicts a radius-height cross section of the azimuthally averaged hydrometeor mixing ratio, tangential velocity, and radial velocity, which are temporally averaged from $t=48$ to $54 \mathrm{~h}$. Figure $13 \mathrm{~d}$ depicts a radius-time cross section of azimuthally averaged tangential velocity at $z=$ $2 \mathrm{~km}$ and diabatic heating rate that is averaged vertically from $z=2$ to $10 \mathrm{~km}$. The tangential velocity intensifies with decreasing RMW to $t=45 \mathrm{~h}$, and then the RMW is approximately constant. A notable point is that the tangential velocity field expands with time after the TC achieves the maximum intensity. The temporal changes in structure and intensify during the SEF event are consistent with observations. 
(a)
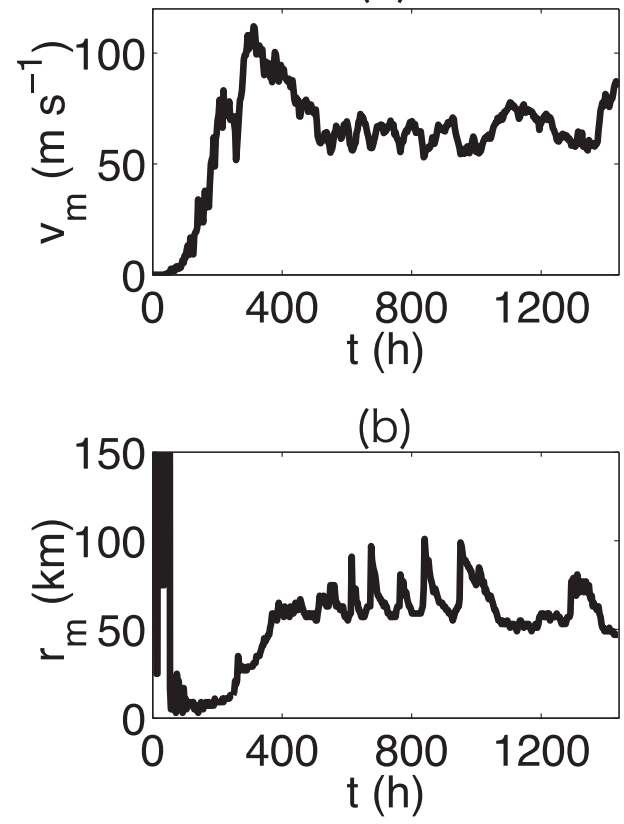
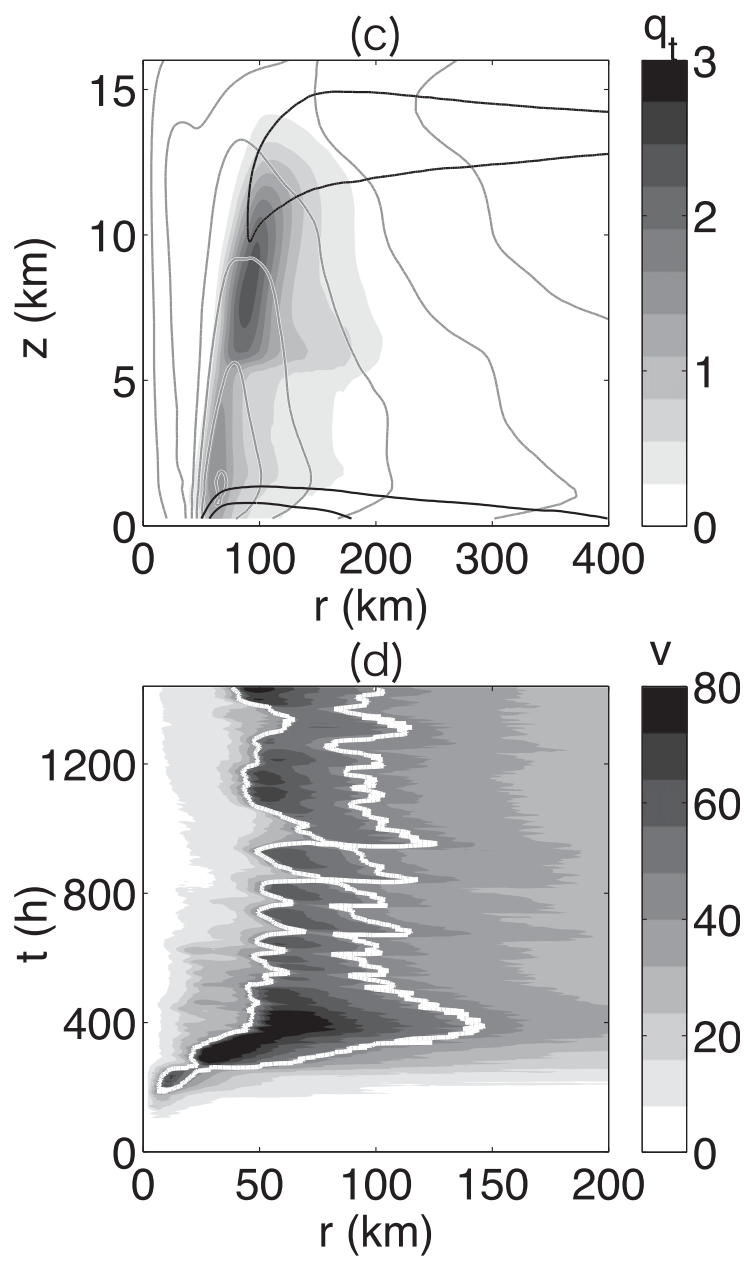

FIG. 11. Time series of (a) TC intensity and (b) RMW of the simulated TC in an axisymmetric full-physics model, (c) radius-height cross section of tangential velocity (gray contours), radial velocity (black contours), and mixing ratio of hydrometeors (shaded) averaged during the quasi-steady state, and (d) radius-time cross section of tangential velocity at $z=2 \mathrm{~km}$. The contour intervals of tangential velocity and radial velocity in (c) are 10 and $5 \mathrm{~m} \mathrm{~s}^{-1}$, respectively. The contour of $50 \mathrm{~m} \mathrm{~s}^{-1}$ is highlighted by the white line in (d).

Figure 14a displays a radius-time cross section of the deviation of tangential velocity from the average between $t=48$ and $96 \mathrm{~h}$, diabatic heating averaged vertically from $z=1$ to $10 \mathrm{~km}$, and $\Upsilon$. The velocity field is smoothed by the $1-2-1$ filter for 20 times before $Y$ is calculated. Only the contours of 1.0 and 2.0 are shown for $\Upsilon$. The velocity deviation increases (i.e., a secondary peak of tangential velocity forms) around $r=125 \mathrm{~km}$ from $t=-17 \mathrm{~h}(t=59 \mathrm{~h}$ of simulation time $)$. The secondary peak intensifies while proceeding inward, which is accompanied with strong diabatic heating. The rapid inward motion of the secondary peak is consistent with Kepert (2017). It suggests that the convectionconvergence feedback plays a role in intensifying the secondary peak of tangential velocity. The time of secondary-peak formation is approximately the same as the increase in diabatic heating in the outer region. The point is that $\Upsilon$ increases from $t-t_{\mathrm{SEF}}=-18 \mathrm{~h}$, which is $1 \mathrm{~h}$ before the formation of a secondary peak.

Figure $14 \mathrm{~b}$ shows a times series of $\Upsilon, \Xi$, and radial gradient of vorticity, which are averaged between $r=$ 115 and $135 \mathrm{~km}$ where the secondary peak forms; $\Upsilon$ is large enough to satisfy the unstable condition from 19 to $15 \mathrm{~h}$ before the time of SEF and is otherwise smaller than that satisfying the condition. The increase in $Y$ results from enhanced $\Xi$ and the radial gradient of vertical vorticity. The velocity field is expanding in this period (cf. Fig. 13d), indicating that the angular velocity is large, which is favorable to increase $\Xi$. In conclusion, a secondary peak of tangential velocity forms $17 \mathrm{~h}$ before the time of SEF, and $Y$ is above the criteria of instability $18 \mathrm{~h}$ before the time of SEF. 

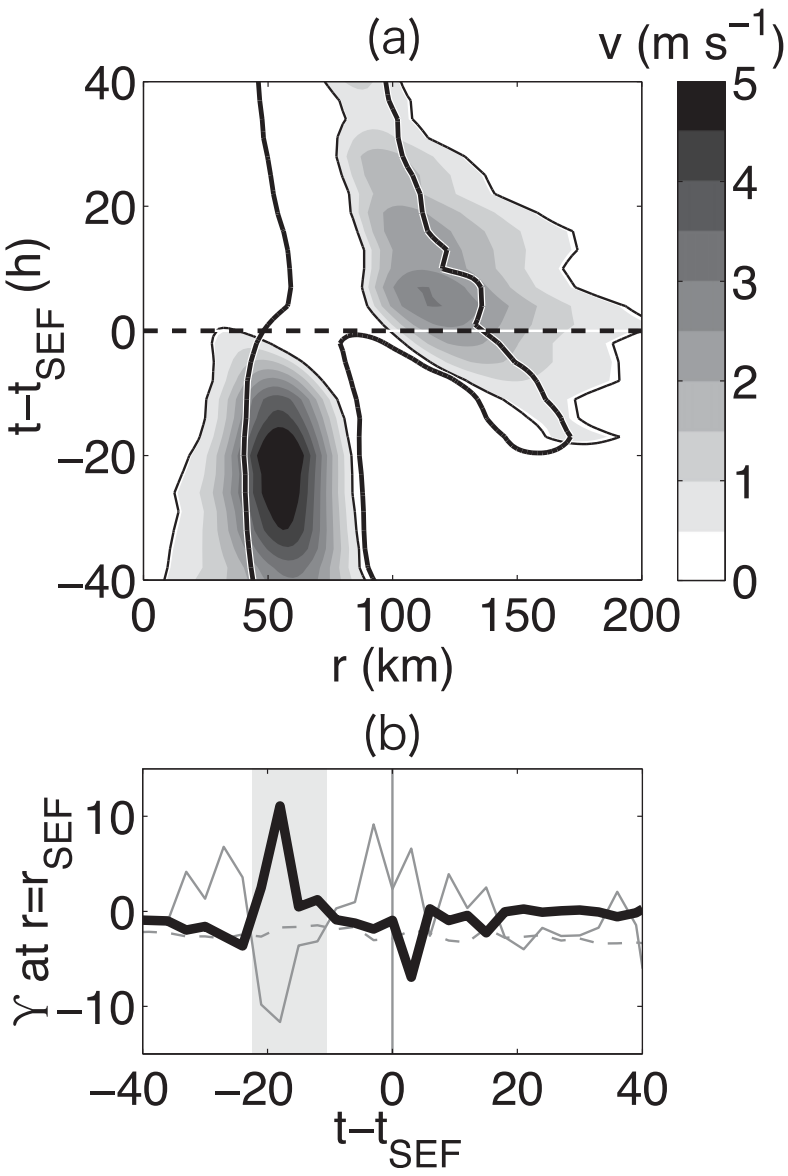

FIG. 12. (a) Radius-time cross section of composited tangential velocity subtracted from the temporal average from $t=-40$ to $40 \mathrm{~h}$ (shaded) and $\Upsilon$ (thick contour). The contour level of $1.0 \mathrm{~m} \mathrm{~s}^{-1}$ for the tangential velocity deviation is highlighted by the thin black line. The contour level of 1.0 is only shown for $\Upsilon$. (b) Time series of $\Upsilon$ (black solid line), $\Xi$ (gray dashed line), and radial gradient of vertical vorticity $\partial_{r} \zeta_{a}$ (gray solid line), which are averaged from $r=$ 135 to $160 \mathrm{~km}$. The horizontal axis shows the time subtracted by the time of SEF.

\section{Discussion}

The theory indicates that a small gradient of vorticity can produce large Ror such that the unstable condition for the linear instability is satisfied. Both Kepert (2013) and the present study show that vertical velocity can be strengthened at a radius where the radial gradient of vorticity is strong. However, the initial radial gradient of vorticity considered in Kepert (2013) is more than 10 times stronger than that in the present study. This study suggests that the vertical velocity can be amplified by the proposed instability under a much smaller gradient of vorticity than used by Kepert (2013) if the vorticity gradient increases at a radius where $\Xi=\bar{\Omega} / \bar{\zeta}_{a}$ is large. Once the vertical velocity and radial vorticity gradient increase up to the magnitude of Kepert (2013), the outer eyewall can intensify via the interaction between the convection and low-level convergence, as Kepert proposed.

The initial amplification of the radial gradient of vorticity to satisfy the unstable condition might be caused by some processes such as diabatic heating in rainbands (Rozoff et al. 2012; Zhang et al. 2016). The present 3D simulation shows little convective organization before the secondary peak of tangential velocity forms (Fig. 14a). Rather, many isolated convective cells are present, but the accumulated effect of these can produce the needed vorticity gradient. Once a secondary peak of tangential velocity forms, a symmetric convective region is organized around that radius. Subsequently, the secondary peak and organized convection may amplify together through the feedback.

Figure 15 illustrates how the proposed feedback mechanism works, with a comparison to the classic Ekman feedback (and damping). Starting from perturbation of vertical velocity with a positive peak, the radial and tangential velocities in the free atmosphere will be produced (cf. Fig. 2). This change will result in faster velocity in the $x$-direction in the boundary layer to the left of the peak of the initial perturbation of vertical velocity and slower velocity to the right. Thus, the responding flow in the boundary layer tends to cause divergence and decrease $w$. On the other hand, when the unstable condition is satisfied, the change in flow fields in the free atmosphere rather produces slower velocity to the left and faster to the right, which increases in $w$. This would happen if the decrease in $\zeta_{a}$ is significant because $u_{b}=-C_{d}|v| v /\left(h_{b} \zeta_{a}\right)$. Furthermore, it is more likely to happen when $\zeta_{a} \gg f$, and if $\zeta_{a} \ll f$, it will not occur.

\section{Conclusions}

We demonstrate an instability via interaction between the free atmosphere and the Ekman layer based on the classic Ekman pumping theory and hypothesize that the very early process of SEF in TCs can be attributed to this instability. The classic Ekman theory indicates that vertical motion at the top of the boundary layer is proportional to the spatial gradient of velocity (relative vorticity), and this results in a decay of velocity perturbations in the free atmosphere. Here, it was hypothesized that once the Ekman pumping velocity is negatively proportional to the velocity gradient under some special conditions, disturbances can grow exponentially.

The instability happens when velocity is fast and changes rapidly in the horizontal direction. Using a 1.5-layer shallow-water model on the $f$ plane [(3)-(7)], we analytically derived an unstable condition (13), in 
(a)

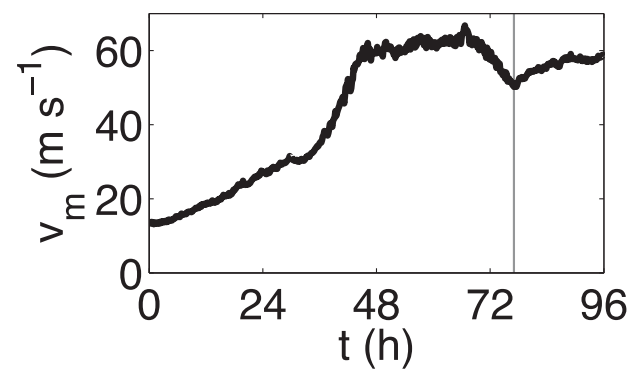

(b)

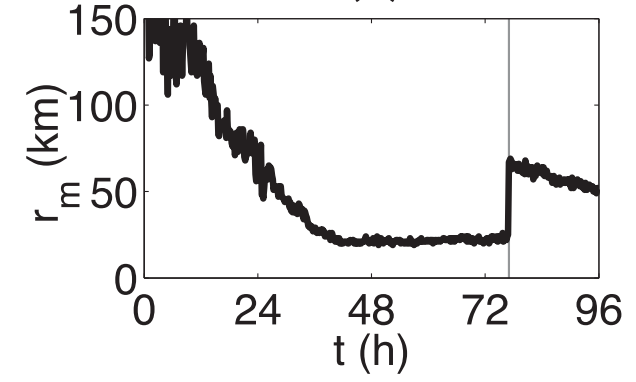

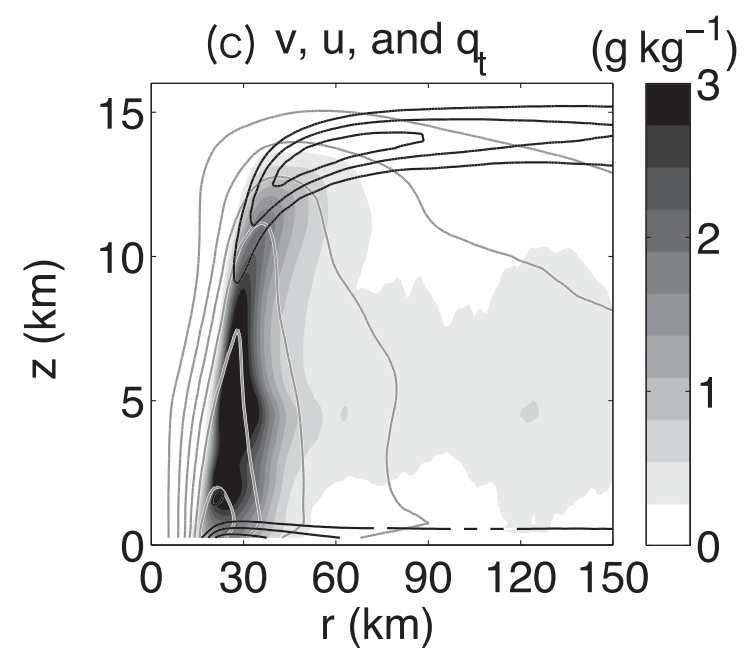

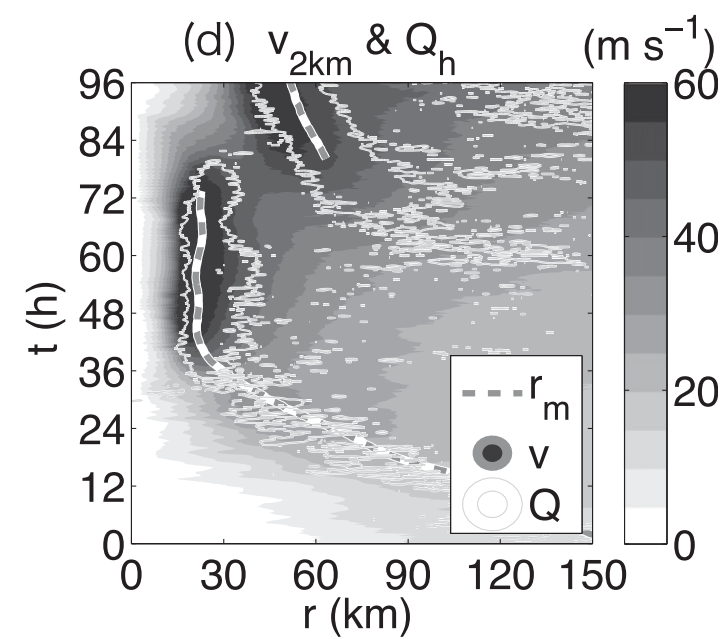

FIG. 13. Time series of (a) TC intensity and (b) RMW of the simulated TC in the WRF Model; (c) radius-height cross section of azimuthally averaged tangential velocity (gray contours), radial velocity (black contours), and mixing ratio of hydrometeors (shaded) averaged from $t=48$ to $54 \mathrm{~h}$; and (d) radius-time cross section of azimuthally averaged tangential velocity at $z=2 \mathrm{~km}$ (shaded), diabatic heating rate vertically averaged from $z=1$ to $10 \mathrm{~km}$ (gray contours), and RMW (gray dashed lines). The contour intervals of tangential velocity, radial velocity, and hydrometeor mixing ratio in (c) are 10 and $5 \mathrm{~m} \mathrm{~s}^{-1}$ and $5 \mathrm{~g} \mathrm{~kg}^{-1}$, respectively. The contour level of $5 \mathrm{~K} \mathrm{~h}^{-1}$ is shown in $(\mathrm{d})$.

which the proportionality coefficient of relative vorticity perturbation in the $w^{\prime}$ equation is negative; that is, $w \propto-\partial_{x} v^{\prime}$. In particular, the unstable condition is more likely to be satisfied for larger Ro, faster basic-state velocity $\bar{v}$, smaller magnitude of negative first-order derivative of $\bar{v}$, and larger magnitude of second-order derivative of $\bar{v}$ provided that $\bar{v}$ is greater than 0 .

Eigenvalue analyses were performed for the linearized equation set. A positive eigenvalue (growth rate) was obtained, and the eigenvector for the maximum eigenvalue showed a velocity field consistent with the hypothesis. In the parameter space of Ro and $\Upsilon$ that controls the unstable condition, both stable and unstable regimes were obtained. The theoretical criteria of instability explains well the border of the unstable regime. Numerical integrations of a nonlinear equation set showed consistent results with the eigenvalue analyses.

We extended the theory to cylindrical coordinates. By linearizing the nondimensionalized equations, it was shown that the instability occurs where $\operatorname{Ro}\left(r \partial_{r} \Xi+2 \Xi\right)$ is greater than 1 . The unstable condition is more likely satisfied for larger Ro, $\Xi$, and $r \partial_{r} \Xi$. Eigenvalue analyses of the linearized 1.5-layer system were performed for varying parameters for the basic-state vortex. The eigenmodes of velocity were consistent with the proposed theory. 

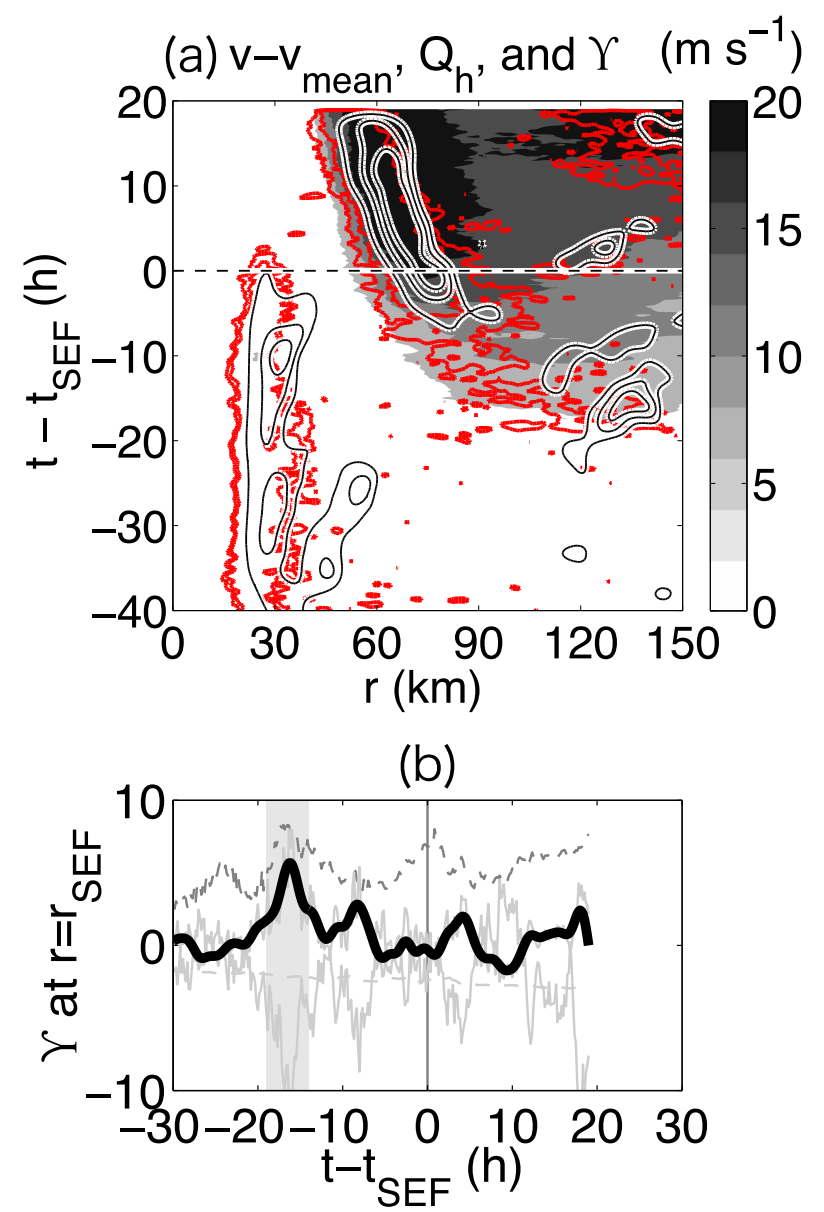

FIG. 14. (a) Radius-time cross section of azimuthally averaged tangential velocity at $z=2 \mathrm{~km}$ subtracted by the temporal average from $t=45$ to $55 \mathrm{~h}$ of $v_{\text {mean }}$, diabatic heating $Q_{h}$ averaged from $z=$ 1 to $10 \mathrm{~km}$ (red contours), and $\Upsilon$ (black contours); $\Upsilon$ is estimated from tangential velocity field smoothed by conducting $1-2-1$ average for 20 times. The contour level of $5.0 \mathrm{~K} \mathrm{~h}^{-1}$ for the diabatic heating rate is shown, whereas the contour interval is 1.0 for $r$. (b) Time series of $\Upsilon$ (black solid line), $\Xi$ (gray dashed line), and radial gradient of vertical vorticity $\partial_{r} \zeta_{a}$ (gray solid line), which are averaged from $r=115$ to $135 \mathrm{~km}$.

The hypothesis was tested by computing the dimensional values of $\Upsilon$ in two different full-physics model simulations. We performed a long-term simulation using the axisymmetric version of CM1, and several cases of SEF and eyewall replacement cycle were successfully simulated. By extracting SEF events and compositing them, it was shown that a secondary peak of tangential velocity formed around 2.5-3.0 times the RMW $22.5 \mathrm{~h}$ before SEF, which was defined as the time when the tangential velocity at the secondary peak is stronger than the primary peak. The value of $\Upsilon$ increased around this radius immediately before the secondary peak formed. The enhancement of $\Upsilon$ resulted from an increasing radial gradient of vorticity with large $\Xi=\bar{\Omega} / \bar{\zeta}_{a}$. (a) Classic theory

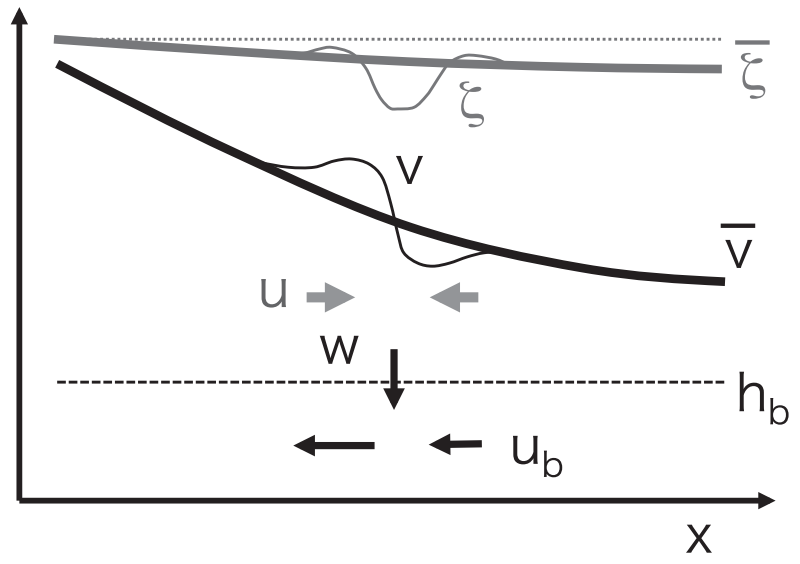

(b) Proposed theory

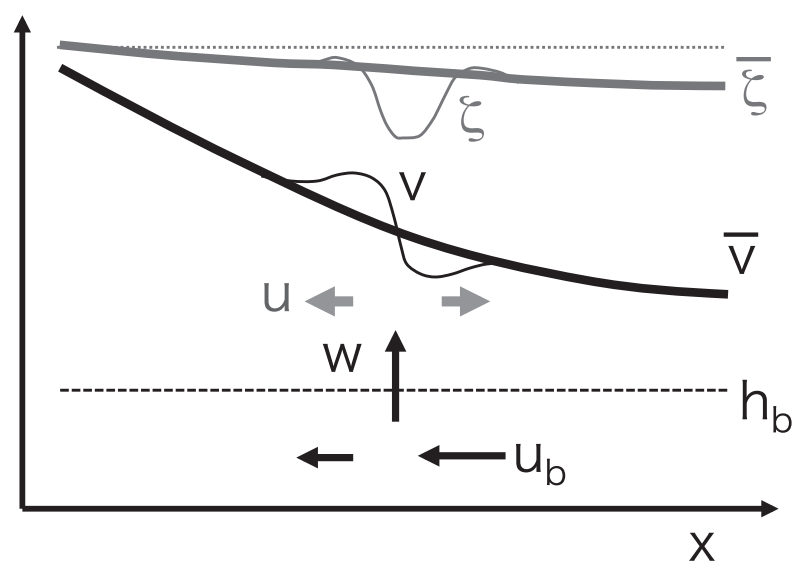

FIG. 15. Schematic of proposed mechanism. (a) Divergent flow caused by $w$ increases $v$ to left and decreases $v$ to right, which results in an decrease in $u_{b}$ to left and an increase in $u_{b}$ to right. Then $w$ decreases. (b) Tendency of $v$ is the same, but $u_{b}$ can increase to right instead of decrease, and then $w$ increases. Since $u_{b}=-C_{d}|v| v /\left(h_{b} \zeta_{a}\right)$, this would happen if $\zeta_{a}$ decreases enough. If $\zeta_{a} \ll f$, this cannot work. Rather, if $\zeta_{a} \gg f$, the $\zeta_{a}$ can change enough for feedback.

Another SEF was simulated using WRF in an idealized environment with nonzero shear. A secondary peak of tangential velocity formed around 5.0-5.5 times the RMW and intensified while proceeding inward, while accompanied by strong convective heating. The local value of $\Upsilon$ increased several hours before the formation of the secondary wind maximum.

The two modeling results suggest that the initial process of SEF, the formation of a secondary peak of a tangential velocity, may be attributed to the instability, and thus $r$ derived from the linear theory can potentially be used as a predictor of SEF. Once the secondary peak is amplified by the mechanism, the convection-convergence 
feedback proposed by Kepert (2013) works to develop the secondary eyewall. Whereas the simulations were conducted by two different models, further verification would be needed using especially observational data as well as other real-case simulations.

Acknowledgments. Y. Miyamoto was supported by JSPS Scientific Research 26-358 for the JSPS fellowship program for overseas researchers. D. S. Nolan was supported by the NSF through Grant AGS-1654831. This work was supported by Keio University Academic Development Funds for Joint Research. The authors thank Dr. Jeff Kepert and two anonymous reviewers for their careful reviews and Drs. Hiroshi Taniguchi and Shigenori Otsuka for fruitful discussions.

\section{APPENDIX A}

\section{Equations for the One-Dimensional 1.5-Layer Shallow-Water System in Cartesian Coordinates}

This appendix describes the governing equations for the one-dimensional, 1.5-layer shallow-water system on an $f$ plane (cf. Fig. 1) considered in the main text. For the $x$ direction that is orthogonal to the direction with fast flow speed, fluid depth and velocity are in geostrophic wind balance in both the free atmosphere and the boundary layer. A bulk drag formula is used to represent the surface friction. No internal diffusion is included in either layers. The mass and momentum conservation equations for the free atmosphere are

$$
\begin{aligned}
\frac{\partial h^{*}}{\partial t^{*}} & =-\frac{\partial h^{*} u^{*}}{\partial x^{*}}+w^{*}, \\
g^{*} \frac{\partial h^{*}}{\partial x^{*}} & =f^{*} v^{*}, \\
\frac{\partial v^{*}}{\partial t^{*}} & =-u^{*} \zeta_{a}^{*},
\end{aligned}
$$

and those for the boundary layer are

$$
\begin{aligned}
-\frac{\partial h_{b}^{*} u_{b}^{*}}{\partial x^{*}} & =w^{*}, \\
u_{b}^{*} \zeta_{a}^{*} & =-\frac{C_{D}\left|v^{*}\right| v^{*}}{h_{b}^{*}},
\end{aligned}
$$

where the asterisk indicates dimensional quantities, $t^{*}$ is the time, $x^{*}$ is the direction in which the fluid depth varies, $y^{*}$ is orthogonal to $x^{*}, h^{*}$ is the fluid depth, $u^{*}$ and $v^{*}$ are the velocities in $x$ and $y$ directions, $w^{*}$ is the vertical velocity at the top of boundary layer, $g^{*}$ is the gravitational acceleration, $f^{*}$ is the Coriolis parameter, $\zeta_{a}^{*}=\partial_{x^{*}} v^{*}+f^{*}$ is the absolute vertical vorticity, $C_{D}$ is the drag coefficient, and the subscript $b$ represents the boundary layer. We have assumed that the $y$-velocity and horizontal gradient of fluid depth in the boundary layer are equal to those in the free atmosphere.

The variables are normalized as

$$
\begin{aligned}
& x^{*}=L x, \quad h^{*}=H h, \quad h_{b}^{*}=H_{b} h_{b}, \quad t^{*}=T t, \\
& u^{*}=\frac{C_{D} V^{2}}{f * H} u, \quad v^{*}=V v, \quad w^{*}=\frac{C_{D} V^{2}}{f * L} w, \\
& u_{b}^{*}=\frac{C_{D} V^{2}}{f * H_{b}} u_{b},
\end{aligned}
$$

where $L, H, T$, and $V$ are the representative scales of length, depth, time, and velocity, respectively. The dimensionless form of the equations is

$$
\begin{aligned}
\frac{1}{\operatorname{Ro}_{1}} \frac{\partial h}{\partial t} & =-\frac{\partial h u}{\partial x}+w, \\
\frac{\operatorname{Ro}}{\operatorname{Fr}^{2}} \frac{\partial h}{\partial x} & =v, \\
\frac{1}{G_{1}} \frac{\partial v}{\partial t} & =-u \zeta_{a},
\end{aligned}
$$

and those for the boundary layer are

$$
\begin{aligned}
-\frac{\partial h_{b} u_{b}}{\partial x} & =w, \\
u_{b} \zeta_{a} & =-\frac{|v| v}{h_{b}},
\end{aligned}
$$

where $\zeta_{a}=\operatorname{Ro} \partial_{x} v+1$ is the dimensionless absolute vertical vorticity, $\mathrm{Ro}=V\left(f^{*} L\right)^{-1}$ is the Rossby number, $\mathrm{Fr}=V\left(g^{*} H\right)^{-1 / 2}$ is the Froude number, and $G_{1}=$ $C_{D} V T / H$.

To obtain the solution for Ekman pumping in the classic theory (e.g., Holton 1992), we linearize the equations by assuming that the basic-state velocity is negligible:

$$
\begin{gathered}
\bar{h} \frac{\partial u^{\prime}}{\partial x}=w^{\prime}, \\
\frac{\operatorname{Ro}}{\operatorname{Fr}^{2}} \frac{\partial h^{\prime}}{\partial x}=v^{\prime}, \\
\frac{1}{G_{1}} \frac{\partial v^{\prime}}{\partial t}=-u^{\prime},
\end{gathered}
$$

and those in the boundary layer are

$$
-\frac{\partial h_{b} u_{b}^{\prime}}{\partial x}=w^{\prime},
$$




$$
-u_{b}^{\prime}=\frac{2|\bar{v}|}{h_{b}} v^{\prime}
$$

where the bar and prime indicate the basic state and perturbation, respectively. Combining (A12) and (A14) yields the tendency equation for $\zeta^{\prime}=\partial_{x} v^{\prime}$ :

$$
\frac{\bar{h}}{G_{1}} \frac{\partial}{\partial t}\left(\frac{\partial v^{\prime}}{\partial x}\right)=-w^{\prime}
$$

On the other hand, substitution of (A15) into (A16) results in a diagnostic equation for $w^{\prime}$ :

$$
w^{\prime}=2|\bar{v}| \frac{\partial v^{\prime}}{\partial x} .
$$

As a result, we obtain the tendency equation for the perturbation vorticity $\zeta^{\prime}=\operatorname{Ro}_{x} v^{\prime}$ :

$$
\frac{\partial \zeta^{\prime}}{\partial t}=-\frac{2 G_{1}|\bar{v}|}{\bar{h}} \zeta^{\prime}
$$

The equation can be integrated, and the solution is

$$
\zeta^{\prime}=\zeta_{0} \exp \left(-\frac{2 G_{1}|\bar{v}|}{\bar{h}} t\right)
$$

where $\zeta_{0}$ is initial value of relative vorticity perturbation. The decaying time scale of vorticity perturbation is short as the effect of surface friction is large. Since the semislip boundary condition is applied, faster velocity results in stronger friction.

\section{APPENDIX B}

\section{Condition for the Instability in Cartesian Coordinates}

We here examine the condition for the instability in Cartesian coordinates, which is $B_{2}<0$, where $B_{2}$ is defined in (10). The condition can be written as

$$
\bar{v} \frac{\partial^{2} \bar{v}}{\partial x^{2}} \operatorname{Ro}^{2}+\frac{\partial \bar{v}}{\partial x} \operatorname{Ro}+1<0,
$$

which is identical to (12). The points of contact on the Ro axis of the curve defined by the lhs can be obtained as

$$
\begin{aligned}
& \mathcal{R}_{1}=\frac{-\partial_{x} \bar{v}+\left[\left(\partial_{x} \bar{v}\right)^{2}-4 \bar{v} \partial_{x}^{2} \bar{v}\right]^{1 / 2}}{2 \bar{v} \partial_{x}^{2} \bar{v}}, \\
& \mathcal{R}_{2}=\frac{-\partial_{x} \bar{v}-\left[\left(\partial_{x} \bar{v}\right)^{2}-4 \bar{v} \partial_{x}^{2} \bar{v}\right]^{1 / 2}}{2 \bar{v} \partial_{x}^{2} \bar{v}} .
\end{aligned}
$$

We need to consider several different cases depending on the sign of coefficients of the first and second terms, whereas the number of cases can be reduced since Ro $>0$. First, when the coefficient of the first term in (B1), $\bar{v} \partial_{x}^{2} \bar{v}$, is positive, the inequality (B1) is satisfied only when the coefficient of the second term $\partial_{x} \bar{v}$ is negative. In this case, the unstable range is limited in $\mathcal{R}_{2}<$ Ro $<\mathcal{R}_{1}$, because the lhs of (B1) is concave upward. When the second term is positive, both the points of contact, $\mathcal{R}_{2}$ and $\mathcal{R}_{1}$, are negative, and hence, Ro must be negative to satisfy (B1), which is not physical. In contrast, when the coefficient of the first term $\bar{v} \partial_{x}^{2} \bar{v}$ is negative, the lhs is concave downward and $\mathcal{R}_{1}<0<\mathcal{R}_{2}$. Hence, the unstable range is much wider $\left(\mathcal{R}_{2}<\mathrm{Ro}\right)$. More specifically, the unstable range is wider when the sign of the coefficient of the second term $\partial_{x} \bar{v}$ is negative compared with when the sign is positive. The points of contact on the Ro axis $\mathcal{R}_{2}$ when the sign is positive and negative correspond to $\mathcal{R}_{1}$ and $\mathcal{R}_{2}$ of the first case with positive coefficient of $\bar{v} \partial_{x}^{2} \bar{v}$, respectively. In conclusion, the inequality is more likely satisfied when both the coefficients, $\bar{v} \partial_{x}^{2} \bar{v}$ and $\partial_{x} \bar{v}$, are negative. In fact, the eigenvalue analysis shows that the eigenmode of $w^{\prime}$ has a maximum where $\bar{v} \partial_{x}^{2} \bar{v}<0$ and $\partial_{x} \bar{v}<0$ (cf. Fig. 3).

\section{APPENDIX C}

\section{Test Calculations for the Instability}

To verify the hypothesized sensitivity of solutions to the coefficient $B_{2}$, some test calculations were performed by numerically integrating the equations for the free atmosphere [(3)-(5)] and vertical velocity [(8)] by artificially setting the coefficients $B_{1}, B_{2}$, and $B_{3}$. For the calculations, the equations were combined into a single diagnostic equation for $v^{\prime}$. Then the equations were discretized in time and space on a staggered grid system. The third-order Runge-Kutta method was used for time integration. The spatial derivatives were discretized by the fourth-order central difference scheme. The periodic boundary condition was applied to the lateral boundary. The domain width was 8 with 201 grid points and uniform grid spacing of 0.04 . The integration time was 150 with the time step of 0.001 .

The initial perturbation horizontal velocities were zero everywhere at the initial time. White noise perturbations with amplitude of 1.0 were added to the vertical velocity. Given $w^{\prime}, u^{\prime}$ was diagnosed from the combined equation using the Gauss-Seidel method. The obtained $u^{\prime}$ was used to calculate temporal change in $\bar{v}$ in (5). Then $w^{\prime}$ was obtained by $\bar{v}$ using (8). The series of calculations was repeated until the end of integration.

The present hypothesis indicates that an instability occurs when $B_{2}$ is negative. Hence, we artificially set one of the three coefficients in (9)-(11) to \pm 1 and the other two to 0 rather than the values determined explicitly 
from velocity distribution. All combinations for the coefficients were tested. Figure $\mathrm{C} 1$ shows a time series of maximum vertical velocity in the test calculations. The experiment with $\left[B_{1}, B_{2}, B_{3}\right]=[0,-1,0]$ is the only one that has an exponentially growing solution. The flow field (not shown) implies that the vertical velocity is amplified through the mechanism described above. In the other calculations, perturbations decay after the integration is initiated. The results show the presence of the linear instability when $B_{2}$ is negative.

\section{APPENDIX D}

\section{Equations for the Rapidly Rotating, Axisymmetric, 1.5-Layer Shallow-Water System}

This appendix introduces the governing equations of a rapidly rotating, axisymmetric, 1.5-layer shallow-water system considered in this study. The mass and momentum conservation equations for the free atmosphere in cylindrical coordinates are given by

$$
\begin{aligned}
\frac{\partial h^{*}}{\partial t^{*}} & =-\frac{1}{r^{*}} \frac{\partial r^{*} u^{*} h^{*}}{\partial r^{*}}+w^{*}, \\
g^{*} \frac{\partial h^{*}}{\partial r^{*}} & =\left(\frac{v^{*}}{r^{*}}+f\right) v^{*}, \\
\frac{\partial v^{*}}{\partial t^{*}} & =-u^{*} \zeta_{a}^{*} .
\end{aligned}
$$

In the boundary layer, we assume that the tangential velocity is the same as that in the free atmosphere (i.e., $\left.v_{b}^{*}=v^{*}\right)$, and a bulk aerodynamic formula is used as the surface stress. The equations are

$$
\begin{aligned}
-\frac{1}{r^{*}} \frac{\partial r u_{b}^{*} h_{b}^{*}}{\partial r^{*}} & =w^{*}, \\
u_{b}^{*} \zeta_{a}^{*} & =-\frac{C_{D}\left|v^{*}\right| v^{*}}{h_{b}^{*}},
\end{aligned}
$$

where $t^{*}$ is the time, $r^{*}$ is the radial direction, $h^{*}$ is the fluid depth, $u^{*}$ and $v^{*}$ are the radial and tangential velocities, $w^{*}$ is the vertical velocity at the top of boundary layer, $g^{*}$ is the gravitational acceleration, $f^{*}$ is the Coriolis parameter, $\zeta_{a}^{*}=\partial_{r^{*}} v^{*}+v^{*} / r^{*}+f$ is the absolute vertical vorticity, $C_{D}$ is the drag coefficient, the subscript $b$ represents the boundary layer, and the asterisk indicates dimensional quantities. Equation (D5) indicates the balance between frictional destruction of absolute angular momentum and radial advection. This assumption has been applied by previous studies (Ooyama 1969). The equations are normalized as

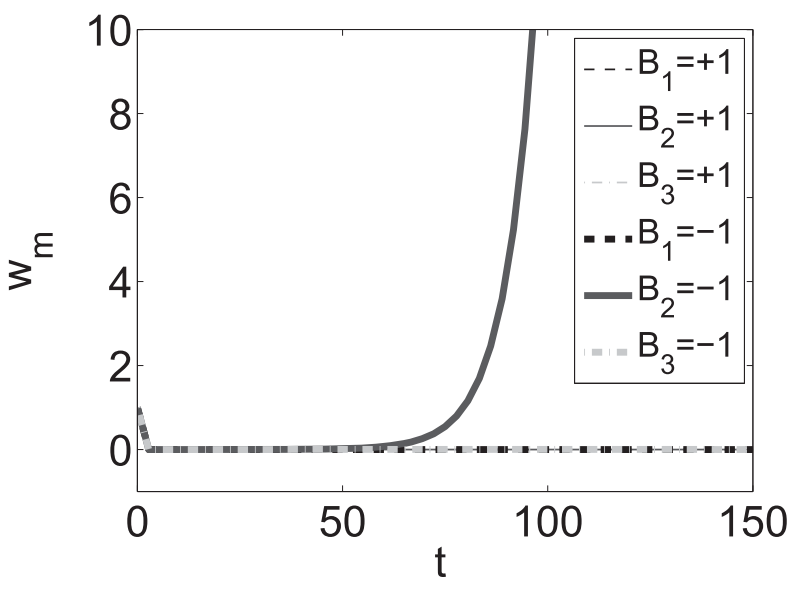

FIG. C1. Time series of domain-maximum $w^{\prime}$ in the test calculations by setting one of the three coefficients in the $w^{\prime}$ equation $[(8)]$ as either $0,+1$, or -1 . In all the calculations, one of the coefficients has a nonzero value (either +1 or -1 ), which is shown in the legend. The thin and bold lines stand for the solutions with the coefficients +1 and -1 . The dashed, solid, and dashed-dotted lines, respectively, represent the solutions with nonzero value for $B_{1}, B_{2}$, and $B_{3}$.

$$
\begin{aligned}
& r^{*}=R r, \quad h^{*}=H h, \quad h_{b}^{*}=H_{b} h_{b}, \quad t^{*}=T t, \\
& u^{*}=\frac{C_{D} V^{2}}{f * H} u, \quad v^{*}=V v, \quad w^{*}=\frac{C_{D} V^{2}}{f * R} w, \\
& u_{b}^{*}=\frac{C_{D} V^{2}}{f * H_{b}} u_{b},
\end{aligned}
$$

where $R, H, H_{b}$, and $T$ are the scales for radius, the depth of free atmosphere, the boundary layer depth, and time, respectively.

The dimensionless equations for the free atmosphere are

$$
\begin{aligned}
\frac{1}{\operatorname{Ro}_{1}} \frac{\partial h}{\partial t} & =-\frac{1}{r} \frac{\partial r u h}{\partial r}+\frac{H_{b}}{H} w, \\
\frac{\operatorname{Ro}}{\operatorname{Fr}^{2}} \frac{\partial h}{\partial r} & =v \xi, \\
\frac{1}{G_{1}} \frac{\partial v}{\partial t} & =-u \zeta_{a},
\end{aligned}
$$

and those for the boundary layer are

$$
\begin{aligned}
-\frac{1}{r} \frac{\partial r u_{b} h_{b}}{\partial r} & =w, \\
\frac{\operatorname{Ro}}{\operatorname{Fr}^{2}} \frac{\partial h}{\partial r} & =v \xi, \\
u_{b} \zeta_{a} & =-\frac{|v| v}{h_{b}},
\end{aligned}
$$


where $\xi=\operatorname{Rov} / r+1, \zeta_{a}=\operatorname{Ro}\left(\partial_{r} v+v / r\right)+1$ is the dimensionless absolute vertical vorticity, $\operatorname{Ro}=V /\left(f^{*} R\right)$ is the Rossby number, $\mathrm{Fr}=V / \sqrt{g^{*} H}$ is the Froude number, and $G_{1}=C_{D} V T / H$.

\section{APPENDIX E}

\section{Details of the WRF Simulation}

The three-dimensional, full-physics simulation used in section $4 \mathrm{~b}$ was produced using the WRF Model, version 3.4.1. The simulation depicts the early development and then rapid intensification of a tropical cyclone in a large, zonally periodic channel using the idealized modeling framework of Nolan (2011), with wind shear balanced by a meridional temperature gradient. However, in many other aspects, the simulation is modeled after the hurricane nature run simulations described by Nolan et al. (2013) and Nolan and Mattocks (2014). The simulation was originally intended to be one of a larger set of idealized simulations used by Koltz and Nolan (2018, manuscript submitted to Wea. Forecasting) for observing system tests, but its robust SEF and ERC made it useful for this paper.

The outer domain has $240 \times 180$ grid points in the zonal and meridional directions with $27-\mathrm{km}$ grid spacing. Three nested, vortex-following grids are used with 9-, 3-, and $1-\mathrm{km}$ grid spacings, with $180 \times 180,360 \times 360$, and $480 \times 480$ grid points, respectively. There are 60 vertical levels between the surface and $20-\mathrm{km}$ altitude. The grid spacings in the WRF vertical coordinate are the same as used in the nature runs (see Fig. 2 of Nolan et al. 2013), and the physical parameterizations are also all identical, including the use of the one-dimensional mixed-layer cooling model of Pollard et al. (1972) available in WRF, version 3.4.1.

The initial vortex and background flow are very similar to the simulations described in Nolan (2011) and Nolan and McGauley (2012). The outer domain is initialized with mean easterly flow of $5 \mathrm{~m} \mathrm{~s}^{-1}$ at the surface, which smoothly increases to $5 \mathrm{~m} \mathrm{~s}^{-1}$ of westerly flow between 850 and $20 \mathrm{hPa}$. An axisymmetric vortex, balanced by temperature and pressure anomalies, is introduced into the eastern end of the domain. The symmetric initial vortex has a maximum tangential wind of $15 \mathrm{~m} \mathrm{~s}^{-1}$ at $r=135 \mathrm{~km}$ and $z=1.5 \mathrm{~km}$. The radial profile of the tangential wind varies as a modified Rankine vortex with decay parameter $a=1 / 3$ and decreases with height using the same analytical function as in (4.2) of Stern and Nolan (2011).

The Coriolis parameter is constant across the channel domain with value $f=5.0 \times 10^{-5} \mathrm{~s}^{-1}$. The SST is set to $28^{\circ} \mathrm{C}$ along the centerline of the domain but varies meridionally. This meridional variation matches the temperature variation that exists at $5-\mathrm{km}$ altitude because of the thermal wind balance. Nolan (2011) used this modification to suppress excessive convection in the northern part of the channel, where otherwise the temperature difference between the SST and the midtroposphere would be substantially increased. The SST variation is approximately linear, ranging from $31.5^{\circ}$ to $24.5^{\circ} \mathrm{C}$ from the south to north edges of the channel.

Finally, as the simulation proceeds, the wind, temperature, and moisture fields on the outer domain are relaxed back to their initial values with a 24-h relaxation time scale. This relaxation does not apply on the nested grids. The relaxation keeps the environmental sounding and wind shear profile around the tropical cyclone roughly constant as the storm develops.

\section{REFERENCES}

Black, M. L., and H. E. Willoughby, 1992: The concentric eyewall cycle of Hurricane Gilbert. Mon. Wea. Rev., 120, 947-957, https://doi.org/10.1175/1520-0493(1992)120<0947: TCECOH $>2.0 . \mathrm{CO} ; 2$.

Bryan, G. H., and J. M. Fritsch, 2002: A benchmark simulation for moist nonhydrostatic numerical models. Mon. Wea. Rev., 130, 2917-2928, https://doi.org/10.1175/1520-0493(2002)130<2917: ABSFMN $>2.0 . \mathrm{CO} ; 2$.

Dai, Y., S. Majumdar, and D. S. Nolan, 2017: Secondary eyewall formation in tropical cyclones by outflow-jet interaction. J. Atmos. Sci., 74, 1941-1958, https://doi.org/10.1175/JAS-D-16-0322.1.

Dodge, P., R. W. Burpee, and F. D. Marks Jr., 1999: The kinematic structure of a hurricane with sea level pressure less than 900 mb. Mon. Wea. Rev., 127, 987-1004, https://doi.org/ 10.1175/1520-0493(1999)127<0987:TKSOAH > 2.0.CO;2.

Ekman, V. W., 1905: On the influence of the Earth's rotation on ocean currents. Arch. Math. Astron. Phys., 2, 1-52.

Eliassen, A., and M. Lystad, 1977: The Ekman layer of a circular vortex: A numerical and theoretical study. Geophys. Norv., 31, $1-16$.

Hakim, G. J., 2011: The mean state of axisymmetric hurricanes in statistical equilibrium. J. Atmos. Sci., 68, 1364-1376, https:// doi.org/10.1175/2010JAS3644.1.

_ 2013: The variability and predictability of axisymmetric hurricanes in statistical equilibrium. J. Atmos. Sci., 70, 9931005, https://doi.org/10.1175/JAS-D-12-0188.1.

Hawkins, H. F., 1983: Hurricane Allen and island obstacles. J. Atmos. Sci., 40, 1360-1361, https://doi.org/10.1175/15200469(1983)040<1360:HAAIO > 2.0.CO;2.

_ Hurricane Inez 1966. Mon. Wea. Rev., 104, 418-442, https:// doi.org/10.1175/1520-0493(1976)104<0418:TSOASI>2.0.CO;2.

Holton, J. R., 1992: An Introduction to Dynamic Meteorology. 3rd ed. Academic Press, 511 pp.

Houze, R. A., Jr., S. S. Chen, B. F. Smull, W.-C. Lee, and M. M. Bell, 2007: Hurricane intensity and eyewall replacement. Science, 315, 1235-1239, https://doi.org/10.1126/science.1135650.

Huang, Y.-H., M. T. Montgomery, and C.-C. Wu, 2012: Concentric eyewall formation in Typhoon Sinlaku (2008). Part II: Axisymmetric dynamical processes. J. Atmos. Sci., 69, 662-674, https://doi.org/10.1175/JAS-D-11-0114.1.

James, I. N., 1995: Introduction to Circulating Atmospheres. Cambridge University Press, 448 pp. 
Jordan, C. L., 1958: Mean soundings for the West Indies area. J. Meteor., 15, 91-97, https://doi.org/10.1175/1520-0469(1958) 015<0091:MSFTWI >2.0.CO;2.

Kepert, J. D., 2013: How does the boundary layer contribute to eyewall replacement cycles in axisymmetric tropical cyclones? J. Atmos. Sci., 70, 2808-2829, https://doi.org/10.1175/JAS-D-13-046.1.

- 2017: Time and space scales in the tropical cyclone boundary layer, and the location of the eyewall updraft. J. Atmos. Sci., 74, 3305-3323, https://doi.org/10.1175/JAS-D-17-0077.1.

— the boundary layer contribute to eyewall replacement cycles in axisymmetric tropical cyclones?"' J. Atmos. Sci., 71, 4692-4704, https://doi.org/10.1175/JAS-D-14-0014.1.

Kossin, J. P., and M. Sitkowski, 2009: An objective model for identifying secondary eyewall formation in hurricanes. Mon. Wea. Rev., 137, 876-892, https://doi.org/10.1175/2008MWR2701.1.

Kuo, H.-C., L.-Y. Lin, C.-P. Chang, and R. T. Williams, 2004: The formation of concentric vorticity structures in typhoons. J. Atmos. Sci., 61, 2722-2734, https://doi.org/10.1175/JAS3286.1.

- W. H. Schubert, C.-L. Tsai, and Y.-F. Kuo, 2008: Vortex interactions and the barotropic aspects of concentric eyewall formation. Mon. Wea. Rev., 136, 5183-5198, https://doi.org/ 10.1175/2008MWR2378.1.

_ C.-P. Chang, Y.-T. Yang, and H. J. Jiang, 2009: Western North Pacific typhoons with concentric eyewalls. Mon. Wea. Rev., 137, 3758-3770, https://doi.org/10.1175/2009MWR2850.1.

Leroux, M.-D., M. Plu, D. Barbary, F. Roux, and P. Arbogast, 2013: Dynamical and physical processes leading to tropical cyclone intensification under upper-level trough forcing. J. Atmos. Sci., 70, 2547-2565, https://doi.org/10.1175/JAS-D-12-0293.1.

Molinari, J., and S. Skubis, 1985: Evolution of the surface wind field in an intensifying tropical cyclone. J. Atmos. Sci., 42, 2865-2879, https://doi.org/10.1175/1520-0469(1985)042<2865: EOTSWF $>2.0 . \mathrm{CO} ; 2$.

— , and D. Vollaro, 1989: External influences on hurricane intensity. Part I: Outflow layer eddy angular momentum fluxes. J. Atmos. Sci., 46, 1093-1105, https://doi.org/10.1175/15200469(1989)046<1093:EIOHIP>2.0.CO;2.

Montgomery, M. T., and R. J. Kallenbach, 1997: A theory for vortex Rossby-waves and its application to spiral bands and intensity changes in hurricanes. Quart. J. Roy. Meteor. Soc., 123, 435-465, https://doi.org/10.1002/qj.49712353810.

Nolan, D. S., 2011: Evaluating environmental favorableness for tropical cyclone development with the method of pointdownscaling. J. Adv. Model. Earth Syst., 3, M08001, https:// doi.org/10.1029/2011MS000063.

— shear: Climatological relationships and physical processes. Cyclones: Formation, Triggers and Control, K. Oouchi and H. Fudeyasu, Eds., Nova Science Publishers, 1-36.

- - and C. A. Mattocks, 2014: Development and evaluation of the second hurricane nature run using the joint OSSE nature run and the WRF Model. 31st Conf. on Hurricane and Tropical Meteorology, San Diego, CA, Amer. Meteor. Soc., 91, https://ams.confex.com/ams/31Hurr/webprogram/ Paper244751.html.

—, R. Atlas, K. T. Bhatia, and L. R. Bucci, 2013: Development and validation of a hurricane nature run using the joint OSSE nature run and the WRF Model. J. Adv. Model. Earth Syst., 5, 382-405, https://doi.org/10.1002/jame.20031.

Nong, S., and K. Emanuel, 2003: A numerical study of the genesis of concentric eyewalls in hurricanes. Quart. J. Roy. Meteor. Soc., 129, 3323-3338, https://doi.org/10.1256/qj.01.132.
Ooyama, K., 1969: Numerical simulation of the life cycle of tropical cyclones. J. Atmos. Sci., 26, 3-40, https://doi.org/10.1175/15200469(1969)026<0003:NSOTLC $>2.0$. CO;2.

Pollard, R. T., P. B. Rhines, and R. O. R. Y. Thompson, 1972: The deepening of the wind-mixed layer. Geophys. Fluid Dyn., $\mathbf{4}$ 381-404, https://doi.org/10.1080/03091927208236105.

Rozoff, C. M., D. S. Nolan, J. P. Kossin, F. Zhang, and J. Fang, 2012: The roles of an expanding wind field and inertial stability in tropical cyclone secondary eyewall formation. J. Atmos. Soc., 69, 2621-2643, https://doi.org/10.1175/JAS-D-11-0326.1.

Skamarock, W. C., and Coauthors, 2008: A description of the Advanced Research WRF version 3. NCAR Tech. Note NCAR/TN475+STR, 123, 113 pp., https://doi.org/10.5065/D68S4MVH.

Stern, D. P., and D. S. Nolan, 2011: On the vertical decay rate of the maximum tangential winds in tropical cyclones. J. Atmos. Sci., 68, 2073-2094, https://doi.org/10.1175/2011JAS3682.1.

Sugimoto, N., K. Ishioka, and S. Yoden, 2007: Balance regimes for the stability of a jet in an $f$-plane shallow water system. Fluid Dyn. Res., 39, 353-377, https://doi.org/10.1016/ j.fluiddyn.2006.07.004.

Sun, Y. Q., Y. Jiang, B. Tan, and F. Zhang, 2013: The governing dynamics of the secondary eyewall formation of Typhoon Sinlaku (2008). J. Atmos. Sci., 70, 3818-3837, https://doi.org/ 10.1175/JAS-D-13-044.1.

Terwey, W. D., and M. T. Montgomery, 2003: Vortex waves and evolution in sharp vorticity gradient vortices. Colorado State University Bluebook 734, $97 \mathrm{pp}$.

_ ized, full-physics modeled hurricanes. J. Geophys. Res., 113, D12112, https://doi.org/10.1029/2007JD008897.

Vallis, G. K., 2017: Atmospheric and Oceanic Fluid Dynamics. Cambridge University Press, 964 pp.

Willoughby, H. E., J. A. Clos, and M. B. Shoreibah, 1982: Concentric eyewalls, secondary wind maxima, and the development of the hurricane vortex. J. Atmos. Sci., 39, 395-411, https://doi.org/10.1175/1520-0469(1982)039<0395: CEWSWM $>2.0 . \mathrm{CO} ; 2$.

—, H.-L. Jin, S. J. Lord, and J. M. Piotrowicz, 1984: Hurricane structure and evolution as simulated by an axisymmetric, nonhydrostatic numerical model. J. Atmos. Sci., 41, 1169-1186, https://doi.org/10.1175/1520-0469(1984)041<1169: HSAEAS $>2.0 . \mathrm{CO} ; 2$.

, R. W. R. Darling, and M. E. Rahn, 2006: Parametric representation of the primary hurricane vortex. Part II: A new family of sectionally continuous profiles. Mon. Wea. Rev., 134, 1102-1120, https://doi.org/10.1175/MWR3106.1.

Wu, C.-C., Y.-H. Huang, and G. Lien, 2012: Concentric eyewall formation in Typhoon Sinlaku (2008). Part I: Assimilation of T-PARC data based on the ensemble Kalman filter (EnKF) J. Atmos. Sci., 140, 506-527, https://doi.org/10.1175/MWR-D11-00057.1.

- - - , and Z. Tan, 2016: Secondary eyewall formation in tropical cyclones. Dynamics and Predictability of Large-Scale, High-Impact Weather and Climate Events, Cambridge University Press, 168-175.

Zhang, F., D. Tao, Y. Q. Sun, and J. D. Kepert, 2016: Dynamics and predictability of secondary eyewall formation in sheared tropical cyclones. J. Adv. Model. Earth Syst., 9, 89-112, https:// doi.org/10.1002/2016MS000729.

Zhu, Z., and P. Zhu, 2014: The role of outer rainband convection in governing the eyewall replacement cycle in numerical simulations of tropical cyclones. J. Geophys. Res. Atmos., 119, 8049-8072, https://doi.org/10.1002/2014JD021899. 